\title{
Optimal Detection of Fechner-Asymmetry
}

\author{
Delphine Cassart, Marc Hallin* \\ and \\ Davy PAINDAVEINE ${ }^{\dagger}$ \\ Institut de Recherche en Statistique, E.C.A.R.E.S., and Département de Mathématique \\ Université Libre de Bruxelles, Brussels, Belgium
}

\begin{abstract}
We consider a general class of skewed univariate densities introduced by Fechner (1897), and derive optimal testing procedures for the null hypothesis of symmetry within that class. Locally and asymptotically optimal (in the Le Cam sense) tests are obtained, both for the case of symmetry with respect to a specified location as for the case of symmetry with respect to some unspecified location. Signed-rank based versions of these tests are also provided. The efficiency properties of the proposed procedures are investigated by a derivation of their asymptotic relative efficiencies with respect to the corresponding Gaussian parametric tests based on the traditional Pearson-Fisher coefficient of skewness. Small-sample performances under several types of asymmetry are investigated via simulations.
\end{abstract}

AMS 1980 subject classification : 62M15, 62G35.

Key words and phrases : Skewed densities, Signed-rank tests, Local asymptotic normality, Tests for symmetry.

\section{Introduction.}

\subsection{Testing for symmetry.}

Symmetry is one of the most important and fundamental structural assumptions in statistics, playing a major role, for instance, in the identifiability of location or intercept under nonparametric conditions: see for instance Stein (1956), Beran (1974) and Stone (1975). This importance explains the huge variety of existing testing procedures of the null hypothesis of symmetry in an i.i.d. sample $X_{1}, \ldots, X_{n}$.

Classical tests of the null hypothesis of symmetry - the hypothesis under which $X_{1}-\theta \stackrel{d}{=}$ $-\left(X_{1}-\theta\right)$ for some location $\theta \in \mathbb{R}$, where $\stackrel{d}{=}$ stands for equality in distribution - are based on third-order moments. Let $m_{k}^{(n)}(\theta):=n^{-1} \sum_{i=1}^{n}\left(X_{i}-\theta\right)^{k}$ and $m_{k}^{(n)}:=m_{k}^{(n)}\left(\bar{X}^{(n)}\right)$, where $\bar{X}^{(n)}:=n^{-1} \sum_{i=1}^{n} X_{i}$. When the location $\theta$ is specified, the test statistic is

$$
n^{1 / 2} m_{3}^{(n)}(\theta) /\left(m_{6}^{(n)}(\theta)\right)^{1 / 2},
$$

*Part of this work was completed while Marc Hallin was visiting ORFE and the Bendheim Center for Finance at Princeton University; the financial support of the Bendheim Center is gratefully acknowledged.

${ }^{\dagger}$ Research supported by a Crédit d'Impulsion of the Fonds National de la Recherche Scientifique, Communauté française de Belgique. 
the null distribution of which, under finite sixth-order moments, is asymptotically standard normal. When $\theta$ is unspecified, the classical test is based on the empirical coefficient of skewness $b_{1}^{(n)}:=m_{3}^{(n)} / s_{n}^{3}$, where $s_{n}:=\left(m_{2}^{(n)}\right)^{1 / 2}$ stands for the empirical standard error in a sample of size $n$. More precisely, this test relies on the asymptotic standard normal distribution (still under finite moments of order six) of

$$
n^{1 / 2} m_{3}^{(n)} /\left(m_{6}^{(n)}-6 s_{n}^{2} m_{4}^{(n)}+9 s_{n}^{6}\right)^{1 / 2} .
$$

These tests are generally considered as Gaussian procedures, although they cannot be considered optimal in any Gaussian sense, as asymmetric alternatives clearly do not belong to the Gaussian world.

Now, symmetry typically is a nonparametric hypothesis. Nonparametric tests of symmetry based on empirical distribution functions have been proposed by Butler (1969) (with a test statistic of the Kolmogorov-Smirnov type), by Rothman and Woodrofe (1972), and Hill and Rao (1977) (with a test statistic of the Cramér-von Mises type) - to quote only a few of them. As usual in such context, some arbitrary distance is adopted on the space of distribution functions, but no optimality issues are addressed; consistency rates are nonparametric. The null hypothesis of symmetry on the other hand also enjoys a rich group invariance structure, that should not remain unexploited. Maximal invariance arguments in this context naturally bring signs and signed ranks into the picture. The most popular nonparametric signed-rank tests of symmetry (with respect to a specified location $\theta$ ) are the sign test, based on the binomial distribution of the number of negative signs in a sample of size $n$, and Wilcoxon's signed-rank test, based on the exact or asymptotic null distribution of $S_{W}^{(n)}:=n^{-1 / 2} \sum_{i=1}^{n} s_{i} R_{+, i}^{(n)}$, where $s_{1}, \ldots, s_{n}$ denote the signs, and $R_{+, 1}^{(n)}, \ldots, R_{+, n}^{(n)}$ the ranks of the absolute values, of the deviation $X_{i}-\theta$ in a sample of size $n$. Other signed-rank tests of symmetry have been proposed by McWilliams (1990) and Randles et al. (1980) - where the ranks and signs however are not those of the observations themselves, and are not distribution-free under the null hypothesis.

Again, these tests are not optimal in any satisfactory sense against asymmetry. The Wilcoxon and sign tests actually are locally asymptotically optimal against location shifts, under logistic or double-exponential densities, respectively. The sign test is even completely insensitive to asymmetric alternatives which preserve the median, i.e., do not include any "shift component".

The main objective of this paper is to provide, for the problem of testing for symmetry (that is, $f(x-\theta)=f(\theta-x)$ for all $x$, with specified or unspecified location $\theta$ ), a concept of optimality that coincides with practitioners' intuition, and to construct parametric and signedrank tests achieving such optimality. This requires embedding the null hypothesis of symmetry into adequate families of asymmetric alternatives. One of the simplest families of asymmetric densities has been proposed more than a century ago by Fechner (1897), and was recently revived by Arellano-Valle, Gómez and Quintana (2005). Denote by $f_{1}$ a symmetric (with respect to the origin) standardized density. The $f_{1}$-Fechner family is the three-parameter collection of densities of the form

$$
f_{\theta, \sigma, \xi}(x):=\frac{1}{\sigma}\left[f_{1}\left(\frac{x-\theta}{(1+\xi) \sigma}\right) I[x \leq \theta]+f_{1}\left(\frac{x-\theta}{(1-\xi) \sigma}\right) I[x>\theta]\right]=\frac{1}{\sigma} f_{1}\left(\frac{x-\theta}{\sigma(1-\xi \operatorname{sign}(x-\theta))}\right),
$$

$x \in \mathbb{R}$, indexed by location $(\theta \in \mathbb{R})$, scale $\left(\sigma \in \mathbb{R}_{0}^{+}\right)$, and a skewness parameter $\xi \in(-1,1)$. Clearly, $\xi=0$, in such families, indicates symmetry, $\xi>0$ asymmetry to the left, and $\xi<0$ asymmetry to the right. Fechner families thus provide a simple and easily interpretable model for location, scale, and skewness. 
The families considered by Arellano-Valle et al. (2005) are more general, with densities of the form

$$
\frac{2}{(a(\xi)+b(\xi)) \sigma}\left[f\left(\frac{x-\theta}{b(\xi) \sigma}\right) I[x \leq \theta]+f\left(\frac{x-\theta}{a(\xi) \sigma}\right) I[x>\theta]\right],
$$

where $\xi \mapsto a(\xi)$ is monotone increasing and $\xi \mapsto b(\xi)$ monotone decreasing, with $a(0)=1=b(0)$, so that $\xi=0$ yields symmetry, $\xi>0$ asymmetry to the left, and $\xi<0$ asymmetry to the right. No obvious choice exists for $a(\xi)$ and $b(\xi)$, and Fechner families clearly correspond to the simple "linear" case $a(\xi)=1-\xi$ and $b(\xi)=1+\xi$. Now, if $a(\xi)$ and $b(\xi)$ are smooth enough in a neighborhood of $\xi=0$, they are of the form

$$
a(\xi)=1+a \xi+o\left(\xi^{2}\right) \quad \text { and } \quad b(\xi)=1-b \xi+o\left(\xi^{2}\right),
$$

respectively, where $a>0$ and $b>0$. If left-asymmetry and right-asymmetry are to be treated similarly, one should have $a(-\xi)=b(\xi)$, hence, in (1.4), we should have $a=b$. Locally, in the vicinity of symmetry, thus, the Arellano-Valle families (1.3) coincide with the Fechner family (1.2).

For simplicity, our tests are developed in the context of Fechner families. More precisely, they are optimal, in a local and asymptotic sense, for the null hypothesis that $\xi=0$ (with specified or unspecified $\theta ; \sigma$ in practice is never specified), against alternatives of the form (1.2) with $\xi>0$ (the $\xi<0$ and $\xi \neq 0$ cases readily follow), for some selected $f_{1}$. Since however the Fechner and Arellano-Valle local alternatives coincide, this optimality extends to any Arellano-Valle family of the form (1.3) (the condition $a=b$ is not even required as far as one-sided optimality is concerned).

Of course, we never assume the actual density of the observations to be of any particular "Fechner form", and the tests we are constructing are expected to be valid under as broad as possible a class of symmetric densities $f_{1}$. Their validity however requires undesirably strong distributional assumptions such as finite moments of order four or six, which significantly restricts their applicability. Taking advantage of the group invariance features of the problem, we also construct signed-rank counterparts, which are distribution-free (asymptotically distribution-free in case of an unspecified $\theta$ ), and hence remain valid under much milder distributional assumptions (for the specified $\theta$ case, they are valid without any distributional assumption), while retaining their optimality features at correctly specified densities.

For instance, the optimal parametric Gaussian test, which is locally asymptotically most powerful against local alternatives of the form (1.2) with $\xi>0$ and $f_{1}=\phi_{1}$ (the standardized normal density) for the specified $\theta$ case, is based on the asymptotically standard normal null distribution of

$$
T_{\phi_{1}}^{(n)}(\theta):=\frac{-1}{\sqrt{n \mathcal{J}^{(n)}\left(\phi_{1}\right)}} \sum_{i=1}^{n} s_{i}(\theta)\left(X_{i}-\theta\right)^{2}
$$

where $\mathcal{J}^{(n)}\left(\phi_{1}\right):=n^{-1} \sum_{i=1}^{n}\left(X_{i}-\theta\right)^{4}$. This test requires finite moments of order four. The normal-score signed-rank counterpart of (1.5) rejects the null hypothesis of symmetry with respect to $\theta$ for large values of

$$
\underset{\sim}{T} \phi_{1}^{(n)}(\theta):=\frac{-1}{\sqrt{n \mathcal{J}^{(n)}\left(\phi_{1}\right)}} \sum_{i=1}^{n} s_{i}(\theta)\left(\Phi^{-1}\left(\frac{n+1+R_{+, i}^{(n)}(\theta)}{2(n+1)}\right)\right)^{2},
$$

where $\Phi$ is the standard normal cumulative distribution function, $R_{+, i}^{(n)}(\theta)$ and $s_{i}(\theta)$ stand for the ranks of the absolute values and the signs of the differences $X_{i}-\theta$, and $\mathcal{J}^{(n)}\left(\phi_{1}\right):=$ 
$n^{-1} \sum_{r=1}^{n}\left(\Phi^{-1}\left(\frac{n+1+r}{2(n+1)}\right)\right)^{4}$. This test is asymptotically equivalent, under Gaussian densities, to the Gaussian optimal test based on (1.5); thus, it is also asymptotically most powerful against local alternatives of the form (1.2) with $\xi>0$ and $f_{1}=\phi_{1}$. However, being distribution-free, it remains valid, unlike the test based on (1.5), under arbitrary symmetric densities. Asymptotic relative efficiencies moreover indicate that the signed-rank tests based on (1.6) under most densities (especially the heavy-tailed ones) dominate those based on (1.5), as well as the more traditional ones based on (1.1). These theoretical findings are confirmed by finite sample simulations.

\subsection{Outline of the paper.}

The paper is organized as follows. In Section 2, we briefly describe the Fechner families to be considered throughout, and provide some examples. Section 3.1 establishes the local asymptotic normality result (with respect to location, scale, and the skewness parameter $\xi$ ) that provides the main theoretical tool of the paper. This result allows for developing asymptotically optimal parametric procedures for $\xi$ under specified densities $f_{1}$, when $\theta$ is specified (Section 3.2) or unspecified (Section 3.3). These tests in general do not remain valid under incorrectly specified densities (namely, under $g_{1} \neq f_{1}$ ): in Sections 3.4 and 3.5, we show how they can be modified in order to remain valid under $g_{1}$ while preserving their optimality under $f_{1}$. The particular case of the pseudo-Gaussian tests (optimal under Gaussian densities but valid under non-Gaussian ones) is investigated in Section 3.6, and their links with the classical procedures based on the Fisher coefficient of skewness (see (1.1)) is discussed. In Sections 4.2 and 4.3, we propose nonparametric (signed-rank-based) versions of the optimal procedures defined in Section 3. The unspecified $\theta$ case requires the delicate estimation of a cross-information quantity, an estimation problem which we discuss extensively in Section 4.5. Some special cases (van der Waerden, Wilcoxon and sign-test scores) of the rank-based statistics are derived in Section 4.4. Sections 4.6 and 4.7 provide some asymptotic relative efficiencies and simulation results assessing the performance of the various tests proposed, under various forms of asymmetry. An appendix collects technical proofs.

\section{Fechner families of skewed densities.}

Denote by $\boldsymbol{X}^{(n)}:=\left(X_{1}^{(n)}, \ldots, X_{n}^{(n)}\right), n \in \mathbb{N}$, an array of i.i.d. observations. Throughout, the null hypotheses we are interested in are

- the hypothesis $\mathcal{H}_{\theta}^{(n)}$ of symmetry with respect to some specified location $\theta \in \mathbb{R}$ : for some $\sigma \in \mathbb{R}_{0}^{+}$, the $X_{i}$ 's have a density function $x \mapsto f(x):=\frac{1}{\sigma} f_{1}\left(\frac{x-\theta}{\sigma}\right)$ (all densities are over the real line, with respect to the Lebesgue measure), where the density $f_{1}$ belongs to the class of symmetric standardized densities

$$
\mathcal{F}_{0}:=\left\{f_{1}: f_{1}(-z)=f_{1}(z) \text { and } \int_{-\infty}^{1} f_{1}(z) d z=0.75\right\}
$$

(the scale parameter $\sigma$ thus is not the standard error, but the population median of the absolute deviations $\left|X_{i}-\theta\right|$, which avoids moment assumptions);

- the hypothesis $\mathcal{H}^{(n)}:=\bigcup_{\theta \in \mathbb{R}} \mathcal{H}_{\theta}^{(n)}$ of symmetry with respect to unspecified location. 
As explained in the introduction, efficient testing requires the definition of families of asymmetric alternatives exhibiting some adequate structure, such as local asymptotic normality, at the null. For a selected class of densities enjoying the required regularity assumptions, we therefore are embedding the null hypothesis of symmetry into Fechner families indexed by location $(\theta \in \mathbb{R})$, scale $\left(\sigma \in \mathbb{R}_{0}^{+}\right)$, and a parameter of skewness $\xi \in(-1,1)$. Consider the class $\mathcal{F}_{1}$ of all densities $f_{1}$ in $\mathcal{F}_{0}$ which are absolutely continuous (for some $\dot{f}_{1}, f_{1}\left(z_{0}\right)=\int_{-\infty}^{z_{0}} \dot{f}_{1}(z) d z>0$ for all $z_{0} \in \mathbb{R}$ ) and that, letting $\phi_{f_{1}}:=-\dot{f}_{1} / f_{1}$,

$$
\begin{gathered}
\mathcal{J}\left(f_{1}\right):=\int_{-\infty}^{+\infty} z^{2} \phi_{f_{1}}^{2}(z) f_{1}(z) d z<\infty \\
\mathcal{I}\left(f_{1}\right):=\int_{-\infty}^{+\infty} \phi_{f_{1}}^{2}(z) f_{1}(z) d z<\infty \text {, and } \mathcal{M}\left(f_{1}\right):=\int_{-\infty}^{+\infty}|z| \phi_{f_{1}}^{2}(z) f_{1}(z) d z<\infty .
\end{gathered}
$$

For all $f_{1} \in \mathcal{F}_{0}$, denote by $\mathrm{P}_{\theta, \sigma, \xi ; f_{1}}^{(n)}$ the probability distribution of $\boldsymbol{X}^{(n)}$ when the $X_{i}$ 's are i.i.d. with density (1.2), a continuous probability density function which is symmetric, left- or rightskewed according as $\xi=0, \xi>0$, or $\xi<0$. The collection $\left\{\mathrm{P}_{\theta, \sigma, \xi ; f_{1}}^{(n)}: \theta \in \mathbb{R}, \sigma \in \mathbb{R}_{0}^{+}, \xi \in(-1,1)\right\}$ will be called the Fechner family associated with $f_{1}$ or $f_{1}$-Fechner family.

Interesting special cases are

(i) the Student Fechner families with $\nu(>0)$ degrees of freedom, associated with $f_{1}(z)=$ $f_{t_{\nu}}(z):=C_{\nu}\left(1+a_{\nu} z^{2} / \nu\right)^{-(1+\nu) / 2} ;$ for $\nu>2, f_{1} \in \mathcal{F}_{1}$, and $\mathcal{I}\left(f_{1}\right)=a_{\nu}(\nu+1) /(\nu+3)$, $\mathcal{J}\left(f_{1}\right)=3(\nu+1) /(\nu+3)$, and $\mathcal{M}\left(f_{1}\right)=4(\nu+1) \sqrt{a_{\nu}} / \sqrt{\nu}(\nu+3) \operatorname{Beta}\left(\frac{\nu}{2}, \frac{1}{2}\right)$, where $\operatorname{Beta}(a, b)$ stands for the Euler Beta function;

(ii) the Gaussian Fechner family, with standardized density $f_{1}(z)=\phi_{1}(z):=\sqrt{a / 2 \pi} \exp \left(-a z^{2} / 2\right)$; $\phi_{1} \in \mathcal{F}_{1}$, and $\mathcal{I}\left(\phi_{1}\right)=a=0.4549, \mathcal{J}\left(\phi_{1}\right)=3$, and $\mathcal{M}\left(\phi_{1}\right)=2 \sqrt{2 a / \pi}$; these values also can be obtained by taking limits, as $\nu \rightarrow \infty$, of the corresponding Student values since $a_{\nu} \rightarrow a$ as $\nu \rightarrow \infty$;

(iii) the logistic Fechner family, with $f_{1}(z)=f_{\text {Log }}(z):=\sqrt{b} \exp (-\sqrt{b} z) /(1+\exp (-\sqrt{b} z))^{2}$; $f_{\text {Log }} \in \mathcal{F}_{1}$, and $\mathcal{I}\left(f_{1}\right)=b / 3, \mathcal{J}\left(f_{1}\right)=\left(12+\pi^{2}\right) / 9$, and $\mathcal{M}\left(f_{1}\right)=\sqrt{b}(2+\ln (16)) / 6$;

(iv) the double-exponential or Laplace Fechner family, with density $f_{1}(z)=f_{\mathcal{L}}(z):=\frac{1}{2 d} \exp (-|z| / d)$; $f_{\mathcal{L}} \in \mathcal{F}_{1}$, and $\mathcal{I}\left(f_{1}\right)=1 / d^{2}, \mathcal{J}\left(f_{1}\right)=2$, and $\mathcal{M}\left(f_{1}\right)=d$

(v) the power-exponential Fechner families, with densities $f_{1}(z)=f_{\mathcal{E}_{\eta}}(z):=C_{\eta} \exp \left(-\left(g_{\eta} z\right)^{2 \eta}\right)$, $\eta>0$; for $\eta>1 / 4, f_{1} \in \mathcal{F}_{1}$, and $\mathcal{I}\left(f_{1}\right)=2 g_{\eta}^{2} \eta \Gamma(2-1 /(2 \eta)) / \Gamma(1+1 /(2 \eta)), \mathcal{J}\left(f_{1}\right)=1+2 \eta$, and $\mathcal{M}\left(f_{1}\right)=2 g_{\eta} \eta / \Gamma(1+1 /(2 \eta))$;

(the positive constants $C_{\nu}, a_{\nu}, a, b, d, C_{\eta}$, and $g_{\eta}$ are such that $f_{1} \in \mathcal{F}_{0}$ ).

Figures 1 and 2 give graphical representations of $(1.2)$ for $f_{1}=\phi_{1}$ and $f_{1}=f_{\mathcal{L}}$, with $\xi=0,0.2,0.4$. In both cases, a positive value of $\xi$ implies a skewness to the left, that increases with the value of $\xi$. 

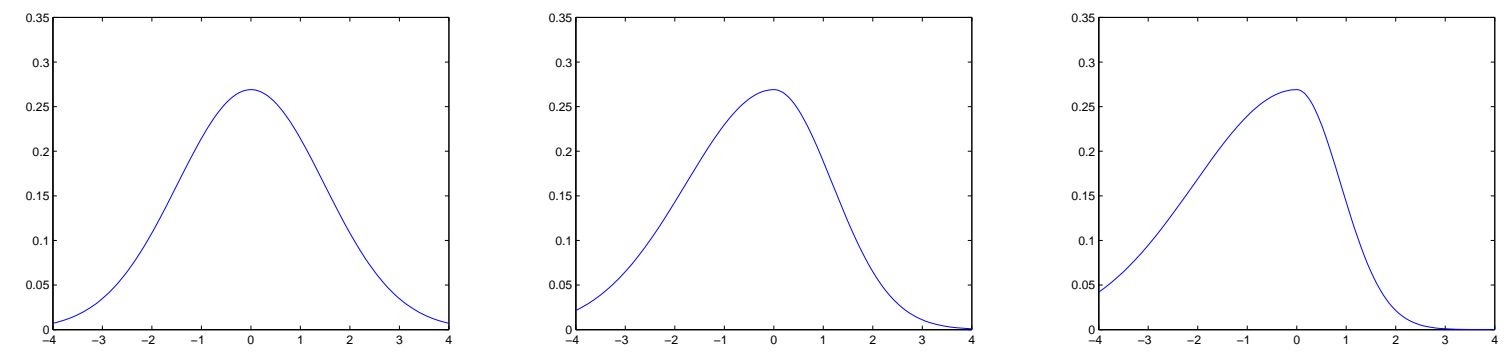

Figure 1: Graphical representation of (1.2) for $f_{1}=\phi_{1}$, with $\xi=0,0.2$, and 0.4.
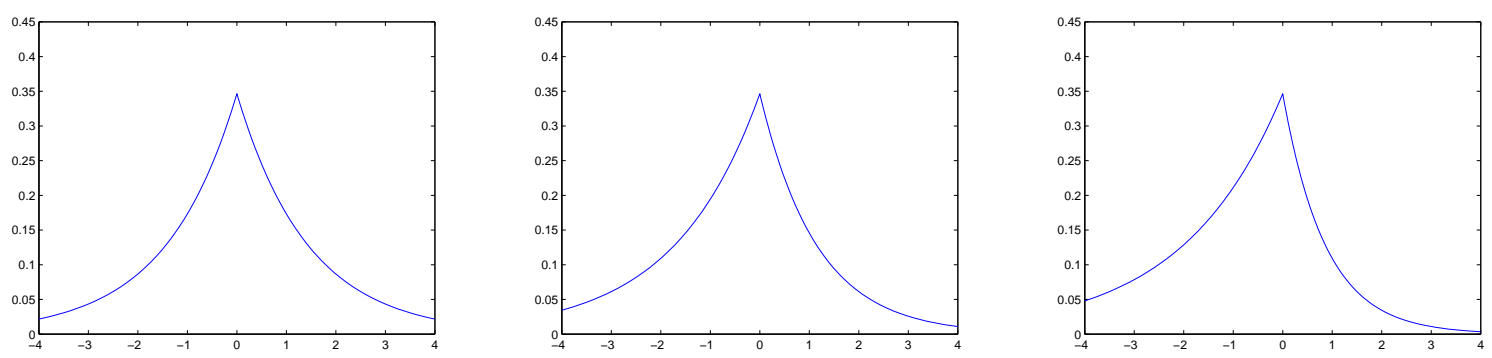

Figure 2: Graphical representation of (1.2) for $f_{1}=f_{\mathcal{L}}$, with $\xi=0,0.2$, and 0.4.

\section{Uniform local asymptotic normality (ULAN) and parametri- cally optimal tests.}

\subsection{ULAN.}

The main technical tool to be used in the sequel is the uniform local asymptotic normality (ULAN), with respect to $\vartheta:=(\theta, \sigma, \xi)^{\prime}$, at $(\theta, \sigma, 0)$, of the Fechner families

$$
\mathcal{P}_{f_{1}}^{(n)}:=\bigcup_{\sigma>0} \mathcal{P}_{\sigma ; f_{1}}^{(n)}:=\bigcup_{\sigma>0}\left\{\mathrm{P}_{\theta, \sigma, \xi ; f_{1}}^{(n)} \mid \theta \in \mathbb{R}, \xi \in(-1,1)\right\}
$$

associated with $f_{1} \in \mathcal{F}_{1}$.

Proposition 3.1 (ULAN). Let $f_{1} \in \mathcal{F}_{1}$. Then the family $\mathcal{P}_{f_{1}}^{(n)}$ is $U L A N$ at any $\vartheta=(\theta, \sigma, 0)^{\prime}$, with (writing $Z_{i}$ for $Z_{i}^{(n)}(\theta, \sigma):=\sigma^{-1}\left(X_{i}^{(n)}-\theta\right)$ ) central sequence

$$
\boldsymbol{\Delta}_{f_{1}}^{(n)}(\boldsymbol{\vartheta})=:\left(\begin{array}{c}
\Delta_{f_{1} ; 1}^{(n)}(\boldsymbol{\vartheta}) \\
\Delta_{f_{1} ; 2}^{(n)}(\boldsymbol{\vartheta}) \\
\Delta_{f_{1} ; 3}^{(n)}(\boldsymbol{\vartheta})
\end{array}\right)=n^{-1 / 2} \sum_{i=1}^{n}\left(\begin{array}{c}
\frac{1}{\sigma} \phi_{f_{1}}\left(Z_{i}\right) \\
\frac{1}{\sigma}\left(\phi_{f_{1}}\left(Z_{i}\right) Z_{i}-1\right) \\
-\phi_{f_{1}}\left(Z_{i}\right)\left|Z_{i}\right|
\end{array}\right)
$$

and full-rank information matrix

$$
\boldsymbol{\Gamma}_{f_{1}}(\boldsymbol{\vartheta})=\left(\begin{array}{ccc}
\sigma^{-2} \mathcal{I}\left(f_{1}\right) & 0 & -\sigma^{-1} \mathcal{M}\left(f_{1}\right) \\
0 & \sigma^{-2}\left(\mathcal{J}\left(f_{1}\right)-1\right) & 0 \\
-\sigma^{-1} \mathcal{M}\left(f_{1}\right) & 0 & \mathcal{J}\left(f_{1}\right)
\end{array}\right)
$$

More precisely, for any $\boldsymbol{\vartheta}^{(n)}:=\left(\theta^{(n)}, \sigma^{(n)}, 0\right)^{\prime}$ such that $\theta^{(n)}-\theta=O\left(n^{-1 / 2}\right)$ and $\sigma^{(n)}-\sigma=$ $O\left(n^{-1 / 2}\right)$, and for any bounded sequence $\boldsymbol{\tau}^{(n)}=\left(t^{(n)}, s^{(n)}, \tau^{(n)}\right)^{\prime} \in \mathbb{R}^{3}$, we have, under $\mathrm{P}_{\boldsymbol{\vartheta}^{(n)} ; f_{1}}^{(n)}$, 
as $n \rightarrow \infty$,

$$
\begin{aligned}
\Lambda_{\boldsymbol{\vartheta}^{(n)}+n^{-1 / 2} \boldsymbol{\tau}^{(n)} / \boldsymbol{\vartheta}^{(n)} ; f_{1}}^{(n)} & :=\log \left(\frac{d \mathrm{P}_{\boldsymbol{\vartheta}^{(n)}+n^{-1 / 2} \boldsymbol{\tau}^{(n)} ; f_{1}}^{(n)}}{d \mathrm{P}_{\boldsymbol{\vartheta}^{(n)} ; f_{1}}^{(n)}}\right) \\
& =\boldsymbol{\tau}^{(n) \prime} \boldsymbol{\Delta}_{f_{1}}^{(n)}\left(\boldsymbol{\vartheta}^{(n)}\right)-\frac{1}{2} \boldsymbol{\tau}^{(n) \prime} \Gamma_{f_{1}}(\boldsymbol{\vartheta}) \boldsymbol{\tau}^{(n)}+o_{\mathrm{P}}(1)
\end{aligned}
$$

and $\boldsymbol{\Delta}_{f_{1}}^{(n)}\left(\boldsymbol{\vartheta}^{(n)}\right) \stackrel{\mathcal{L}}{\rightarrow} \mathcal{N}\left(\mathbf{0}, \Gamma_{f_{1}}(\boldsymbol{\vartheta})\right)$.

See the appendix for a proof.

Note the non-diagonal form of the information matrix $\Gamma_{f_{1}}(\vartheta)$, which implies that location and skewness variations, in Fechner families, are related. The Gaussian versions of (3.2) and (3.3) are

$$
\boldsymbol{\Delta}_{\phi_{1}}^{(n)}(\boldsymbol{\vartheta})=n^{-1 / 2} \sum_{i=1}^{n}\left(\begin{array}{c}
\frac{a}{\sigma} Z_{i} \\
\frac{1}{\sigma}\left(a Z_{i}^{2}-1\right) \\
-a Z_{i}\left|Z_{i}\right|
\end{array}\right) \quad \text { and } \quad \boldsymbol{\Gamma}_{\phi_{1}}(\boldsymbol{\vartheta})=\left(\begin{array}{ccc}
a \sigma^{-2} & 0 & -2 \sigma^{-1} \sqrt{\frac{2 a}{\pi}} \\
0 & 2 \sigma^{-2} & 0 \\
-2 \sigma^{-1} \sqrt{\frac{2 a}{\pi}} & 0 & 3
\end{array}\right)
$$

respectively

\subsection{Optimal parametric tests: specified density, specified location.}

For specified $f_{1} \in \mathcal{F}_{1}$, consider the null hypothesis $\mathcal{H}_{\theta ; f_{1}}^{(n)}:=\bigcup_{\sigma \in \mathbb{R}_{0}^{+}}\left\{\mathrm{P}_{\theta, \sigma, 0 ; f_{1}}^{(n)}\right\}$ of symmetry with respect to some specified location $\theta$. ULAN and the diagonal structure of the covariance matrix corresponding to the $\sigma$ - and the $\xi$-parts of the central sequence, i.e.,

$$
\left(\begin{array}{cc}
\sigma^{-2}\left(\mathcal{J}\left(f_{1}\right)-1\right) & 0 \\
0 & \mathcal{J}\left(f_{1}\right)
\end{array}\right)
$$

imply that substituting discretized root- $n$ consistent estimators $\hat{\sigma}$ for the unknown $\sigma$ has no influence, asymptotically, on the $\xi$-part of the central sequence. Recall that a sequence of estimators $\hat{\lambda}^{(n)}$ defined in a sequence of experiments $\left\{\mathrm{P}_{\lambda}^{(n)} \mid \lambda \in \Lambda\right\}$ indexed by some parameter $\lambda$ is root- $n$ consistent and asymptotically discrete if, under $\mathrm{P}_{\lambda}^{(n)}$, as $n \rightarrow \infty$,

(C1) $\hat{\lambda}^{(n)}-\lambda=O_{\mathrm{P}}\left(n^{-1 / 2}\right)$, and

(C2) the number of possible values of $\hat{\lambda}^{(n)}$ in balls with $O\left(n^{-1 / 2}\right)$ radius centered at $\lambda$ is bounded as $n \rightarrow \infty$.

An estimator $\hat{\lambda}^{(n)}$ satisfying (C1) but not (C2) is easily discretized by letting, for some arbitrary $c>0, \hat{\lambda}_{\#}^{(n)}:=\left(c n^{1 / 2}\right)^{-1} \operatorname{sign}\left(\hat{\lambda}^{(n)}\right)\left\lceil c n^{1 / 2}\left|\hat{\lambda}^{(n)}\right|\right\rceil$, which satisfies both (C1) and (C2). Subscript \# in the sequel indicates such a discretization (the constant $c$ asymptotically plays no role). It is well-known, however, that $(\mathrm{C} 2)$ has no implications in practice, where $n$ is fixed, as $c$ can be chosen arbitrarily large.

It follows that locally asymptotically uniformly most powerful tests of $\mathcal{H}_{\theta ; f_{1}}^{(n)}$ can be based on $\Delta_{f_{1} ; 3}^{(n)}\left(\theta, \hat{\sigma}_{\#}, 0\right)$, hence on $T_{f_{1}}^{(n)}\left(\theta, \hat{\sigma}_{\#}\right)$, where

$$
T_{f_{1}}^{(n)}(\theta, \sigma):=\frac{-1}{\sqrt{n \mathcal{J}\left(f_{1}\right)}} \sum_{i=1}^{n} \phi_{f_{1}}\left(Z_{i}(\theta, \sigma)\right)\left|Z_{i}(\theta, \sigma)\right| .
$$


A consistent estimator of $\sigma$ that does not require any moment assumption is, for instance, the empirical median $\hat{\sigma}:=\operatorname{Med}\left(\left|X_{i}^{(n)}-\theta\right|\right)$ of the absolute deviations of the $X_{i}^{(n)}$ 's from $\theta$.

The following proposition then results from classical results on ULAN families (see, e.g., Chapter 11 of Le Cam 1986).

Proposition 3.2 Let $f_{1} \in \mathcal{F}_{1}$. Then,

(i) $T_{f_{1}}^{(n)}\left(\theta, \hat{\sigma}_{\#}\right)=T_{f_{1}}^{(n)}(\theta, \sigma)+o_{\mathrm{P}}(1)$ is asymptotically normal, with mean zero under $\mathrm{P}_{\theta, \sigma, 0 ; f_{1}}^{(n)}$, mean $\tau\left(\mathcal{J}\left(f_{1}\right)\right)^{1 / 2}$ under $\mathrm{P}_{\theta, \sigma, n^{-1 / 2} \tau ; f_{1}}^{(n)}$, and variance one under both;

(ii) the sequence of tests rejecting the null hypothesis $\mathcal{H}_{\theta ; f_{1}}^{(n)}$ of symmetry (with standardized density $\left.f_{1}\right)$ whenever $T_{f_{1}}^{(n)}\left(\theta, \hat{\sigma}_{\#}\right)$ exceeds the $(1-\alpha)$ standard normal quantile $z_{\alpha}$ is locally asymptotically uniformly most powerful, at asymptotic level $\alpha$, for $\mathcal{H}_{\theta ; f_{1}}^{(n)}$ against $\bigcup_{\xi>0} \bigcup_{\sigma \in \mathbb{R}_{0}^{+}}\left\{\mathrm{P}_{\theta, \sigma, \xi ; f_{1}}^{(n)}\right\}$.

Locally asymptotically maximin two-sided tests are easily derived along the same lines. The Gaussian version of (3.4) is

$$
T_{\phi_{1}}^{(n)}(\theta, \sigma):=\frac{-a}{\sqrt{3 n}} \sum_{i=1}^{n} \operatorname{sign}\left(Z_{i}(\theta, \sigma)\right) Z_{i}^{2}(\theta, \sigma) .
$$

\subsection{Optimal parametric tests: specified density, unspecified location.}

We now consider the unspecified $\theta$ case, that is, the null hypothesis $\mathcal{H}_{f_{1}}^{(n)}:=\bigcup_{\theta \in \mathbb{R}} \bigcup_{\sigma \in \mathbb{R}_{0}^{+}}\left\{\mathrm{P}_{\theta, \sigma, 0 ; f_{1}}^{(n)}\right\}$. The fact that the asymptotic covariance between the $\theta$ - and the $\xi$-part is not zero implies that a local perturbation of location has the same asymptotic impact on $\Delta_{f_{1} ; 3}^{(n)}(\theta, \sigma, 0)$ as a local perturbation of $\xi$. LAN and the convergence of local experiments to the Gaussian shift experiment

$$
\left(\begin{array}{c}
\Delta_{1} \\
\Delta_{3}
\end{array}\right) \sim \mathcal{N}\left(\left(\begin{array}{cc}
\Gamma_{f_{1}, 11}(\boldsymbol{\vartheta}) & \Gamma_{f_{1}, 13}(\boldsymbol{\vartheta}) \\
\Gamma_{f_{1}, 13}(\boldsymbol{\vartheta}) & \Gamma_{f_{1}, 33}(\boldsymbol{\vartheta})
\end{array}\right)\left(\begin{array}{c}
\tau_{1} \\
\tau_{3}
\end{array}\right),\left(\begin{array}{cc}
\Gamma_{f_{1}, 11}(\boldsymbol{\vartheta}) & \Gamma_{f_{1}, 13}(\boldsymbol{\vartheta}) \\
\Gamma_{f_{1}, 13}(\boldsymbol{\vartheta}) & \Gamma_{f_{1}, 33}(\boldsymbol{\vartheta})
\end{array}\right)\right),\left(\tau_{1}, \tau_{3}\right)^{\prime} \in \mathbb{R}^{2}
$$

imply that locally optimal inference on $\xi$, when the location under the null is unspecified, should be based on the residual of the regression (in (3.5)), of $\Delta_{3}$ with respect to $\Delta_{1}$, computed at $\Delta_{f_{1} ; 3}^{(n)}(\theta, \sigma, 0)$ and $\Delta_{f_{1} ; 1}^{(n)}(\theta, \sigma, 0)$. This residual takes the form $\Delta_{3}-\left(\Gamma_{f_{1}, 11}(\boldsymbol{\vartheta})\right)^{-1} \Gamma_{f_{1}, 13}(\boldsymbol{\vartheta}) \Delta_{1}$; the resulting $f_{1}$-efficient central sequence for symmetry is thus (letting $\eta\left(f_{1}\right):=\mathcal{M}\left(f_{1}\right) / \mathcal{I}\left(f_{1}\right)$ )

$$
\Delta_{f_{1}}^{*(n)}(\boldsymbol{\vartheta})=n^{-1 / 2} \sum_{i=1}^{n} \phi_{f_{1}}\left(Z_{i}(\theta, \sigma)\right)\left(\eta\left(f_{1}\right)-\left|Z_{i}(\theta, \sigma)\right|\right) .
$$

This efficient central sequence under $\mathrm{P}_{\boldsymbol{\vartheta} ; f_{1}}^{(n)}$ is asymptotically normal, with mean zero and variance

$$
\gamma_{f_{1}}^{*}:=\mathcal{J}\left(f_{1}\right)-\mathcal{M}^{2}\left(f_{1}\right) / \mathcal{I}\left(f_{1}\right) .
$$

The "cost", in terms of information for skewness, of not specifying $\theta$ in the $f_{1}$-Fechner family is thus $\mathcal{M}^{2}\left(f_{1}\right) / \mathcal{I}\left(f_{1}\right)$. 
It follows that locally asymptotically uniformly most powerful tests of $\mathcal{H}_{f_{1}}^{(n)}$ can be based on $\Delta_{f_{1}}^{*(n)}\left(\hat{\theta}_{\#}, \hat{\sigma}_{\#}, 0\right)$, hence on $T_{f_{1}}^{*(n)}\left(\hat{\theta}_{\#}, \hat{\sigma}_{\#}\right)$, where

$$
T_{f_{1}}^{*(n)}(\theta, \sigma):=\frac{1}{\sqrt{n \gamma_{f_{1}}^{*}}} \sum_{i=1}^{n} \phi_{f_{1}}\left(Z_{i}(\theta, \sigma)\right)\left(\eta\left(f_{1}\right)-\left|Z_{i}(\theta, \sigma)\right|\right),
$$

where $\hat{\theta}_{\#}$ and $\hat{\sigma}_{\#}$ satisfy $(\mathrm{C} 1)$ and $(\mathrm{C} 2)$. Such estimators that do not require any moment assumption can be obtained, for instance, by discretizing the median $\hat{\theta}:=\operatorname{Med}\left(X_{i}^{(n)}\right)$ of the $X_{i}^{(n)}$ 's and the median $\operatorname{Med}\left(\left|X_{i}^{(n)}-\hat{\theta}\right|\right)$ of the absolute deviations $\left|X_{i}^{(n)}-\hat{\theta}\right|$.

The following proposition then follows from classical results on ULAN families (see, e.g., Chapter 11 of Le Cam 1986).

Proposition 3.3 Let $f_{1} \in \mathcal{F}_{1}$. Then,

(i) $T_{f_{1}}^{*(n)}\left(\hat{\theta}_{\#}, \hat{\sigma}_{\#}\right)=T_{f_{1}}^{*(n)}(\theta, \sigma)+o_{\mathrm{P}}(1)$ is asymptotically normal, with mean zero under $\mathrm{P}_{\theta, \sigma, 0 ; f_{1}}^{(n)}$, mean $\tau\left(\gamma_{f_{1}}^{*}\right)^{1 / 2}$ under $\mathrm{P}_{\theta, \sigma, n^{-1 / 2} \tau ; f_{1}}^{(n)}$, and variance one under both;

(ii) the sequence of tests rejecting the null hypothesis $\mathcal{H}_{f_{1}}^{(n)}$ of symmetry (with standardized density $\left.f_{1}\right)$ whenever $T_{f_{1}}^{*(n)}\left(\hat{\theta}_{\#}, \hat{\sigma}_{\#}\right)$ exceeds the $(1-\alpha)$ standard normal quantile $z_{\alpha}$ is locally asymptotically optimal (most stringent), at asymptotic level $\alpha$, for $\mathcal{H}_{f_{1}}^{(n)}$ against $\bigcup_{\xi>0} \bigcup_{\theta \in \mathbb{R}} \bigcup_{\sigma \in \mathbb{R}_{0}^{+}}\left\{\mathrm{P}_{\theta, \sigma, \xi ; f_{1}}^{(n)}\right\}$.

Locally asymptotically maximin two-sided tests are easily derived along the same lines. The Gaussian version of (3.7) is

$$
T_{\phi_{1}}^{*(n)}(\theta, \sigma):=\frac{1}{\sqrt{(3-8 / \pi) n}} \sum_{i=1}^{n} Z_{i}(\theta, \sigma)\left(\sqrt{(8 / a \pi)}-a\left|Z_{i}(\theta, \sigma)\right|\right) .
$$

\subsection{Optimal parametric tests: unspecified density, specified location.}

The parametric tests based on (3.4) and (3.7) achieve local and asymptotic optimality at correctly specified $f_{1}$, which sets the parametric efficiency bounds for the problem, but has limited practical value. These tests indeed in general do not remain valid under density $g_{1} \neq f_{1}$. If they are to be adapted to more realistic null hypotheses $\mathcal{H}_{\theta}^{(n)}:=\bigcup_{g_{1}} \mathcal{H}_{\theta ; g_{1}}^{(n)}$ and $\mathcal{H}^{(n)}:=\bigcup_{g_{1}} \mathcal{H}_{g_{1}}^{(n)}$ of symmetry with unspecified standardized density $g_{1}$ ranging over some broad subfamily of $\mathcal{F}_{1}$, three technical problems have to be treated with care: the centering and scaling of $T_{f_{1}}^{(n)}$ and $T_{f_{1}}^{*(n)}$ under the null, the impact (under $\mathcal{H}^{(n)}$ ) on the asymptotic distribution of $T_{f_{1}}^{*(n)}$ of the substitution of $\hat{\theta}$ for $\theta$, and the impact (under $\mathcal{H}_{\theta}^{(n)}$ and $\mathcal{H}^{(n)}$ ) of the substitution of $\hat{\sigma}$ for $\sigma$.

Let us first assume that both $\theta$ and $\sigma$ are specified. Note that $\Delta_{f_{1} ; 3}^{(n)}(\boldsymbol{\vartheta})$ remains centered under $\mathrm{P}_{\theta, \sigma, 0 ; g_{1}}^{(n)}$. Indeed, the function $z \mapsto \phi_{f_{1}}(z)|z|$ is an odd function, so that the expectation of $\phi_{f_{1}}(Z)|Z|$ is zero for any symmetrically distributed random variable $Z$-provided that such expectation exists. The variance under $\mathrm{P}_{\theta, \sigma, 0 ; g_{1}}^{(n)}$ of $\Delta_{f_{1} ; 3}^{(n)}(\boldsymbol{\vartheta})$ is then

$$
\mathcal{J}_{g_{1}}\left(f_{1}\right):=\mathrm{E}_{g_{1}}\left[\left(\phi_{f_{1}}\left(Z_{i}\right)\left|Z_{i}\right|\right)^{2}\right]=\int_{-\infty}^{\infty} z^{2} \phi_{f_{1}}^{2}(z) g_{1}(z) d z
$$


(still, provided that this integral converges).

We know from Le Cam's third Lemma that, under $\mathrm{P}_{\theta, \sigma, 0 ; g_{1}}^{(n)}$, the impact on $\Delta_{f_{1} ; 3}^{(n)}(\boldsymbol{\vartheta})$ of a perturbation of the scale $\sigma$ depends on the covariance in the joint asymptotically multinormal distribution (still, under $\mathrm{P}_{\theta, \sigma, 0 ; g_{1}}^{(n)}$ ) of $\Delta_{f_{1} ; 3}^{(n)}$ and $\Delta_{g_{1} ; 2}^{(n)}$. Now,

$$
\left(\begin{array}{c}
\Delta_{f_{1} ; 3}^{(n)}(\theta, \sigma, 0) \\
\Delta_{g_{1} ; 2}^{(n)}(\theta, \sigma, 0)
\end{array}\right)=n^{-1 / 2} \sum_{i=1}^{n}\left(\begin{array}{c}
-\phi_{f_{1}}\left(Z_{i}\right)\left|Z_{i}\right| \\
\frac{1}{\sigma}\left(\phi_{g_{1}}\left(Z_{i}\right) Z_{i}-1\right)
\end{array}\right)
$$

under $\mathrm{P}_{\theta, \sigma, 0 ; g_{1}}^{(n)}$ is easily shown to be asymptotically normal, with mean zero and diagonal covariance matrix, since, as the integral of a skew-symmetric function, $\int_{-\infty}^{\infty} \phi_{f_{1}}(z)|z|\left(\phi_{g_{1}}(z) z-\right.$ 1) $g_{1}(z) d z=0$. The effect on the asymptotic distribution of $\Delta_{f_{1} ; 3}^{(n)}$ of a root- $n$ perturbation of $\sigma$ thus is asymptotically nil (in distribution); the asymptotic linearity result of Proposition 6.1 allows for extending (in probability) this conclusion to the perturbations induced by substituting any duly discretized root- $n$ consistent estimator $\hat{\sigma}_{\#}^{(n)}$ for $\sigma$.

If $f_{1} \in \mathcal{F}_{1}$ and $g_{1}$ is such that $\mathcal{J}_{g_{1}}\left(f_{1}\right)<\infty$,

$$
\mathcal{J}^{(n)}\left(\theta, \sigma ; f_{1}\right):=n^{-1} \sum_{i=1}^{n} Z_{i}^{2}(\theta, \sigma) \phi_{f_{1}}^{2}\left(Z_{i}(\theta, \sigma)\right)
$$

under $\mathrm{P}_{\theta, \sigma, 0 ; g_{1}}^{(n)}$, is a consistent estimate of $\mathcal{J}_{g_{1}}\left(f_{1}\right)$. In practice, $\mathcal{J}^{(n)}\left(\theta, \sigma ; f_{1}\right)$ cannot be computed from the observations, and $Z_{i}\left(\theta, \hat{\sigma}_{\#}\right)$ is to be substituted for $Z_{i}(\theta, \sigma)$ in (3.8) yielding $\mathcal{J}^{(n)}\left(\theta, \hat{\sigma}_{\#} ; f_{1}\right)$. This substitution in general requires a slight reinforcement of regularity assumptions. Routine application of Le Cam's third Lemma implies that $\mathcal{J}^{(n)}\left(\theta, \hat{\sigma}_{\#} ; f_{1}\right)-\mathcal{J}^{(n)}\left(\theta, \sigma ; f_{1}\right)$ is $o_{\mathrm{P}}(1)$ under $\mathrm{P}_{\theta, \sigma, 0 ; g_{1}}^{(n)}$ provided that the asymptotic covariance of $\mathcal{J}^{(n)}\left(\theta, \sigma ; f_{1}\right)$ and $\Delta_{g_{1} ; 2}^{(n)}$ is finite. A simple computation shows that a sufficient condition for this is

$$
\int_{-\infty}^{\infty} z^{3} \phi_{f_{1}}^{2}(z) \phi_{g_{1}}(z) g_{1}(z) d z<\infty
$$

Denote by $\mathcal{F}_{f_{1}}$ the subset of $\mathcal{F}_{1}$ for which (3.9) holds and $\mathcal{J}_{g_{1}}\left(f_{1}\right)<\infty$. Defining

$$
\hat{T}_{f_{1}}^{(n)}(\theta, \sigma):=\frac{-1}{\sqrt{n \mathcal{J}^{(n)}\left(\theta, \sigma ; f_{1}\right)}} \sum_{i=1}^{n} \phi_{f_{1}}\left(Z_{i}(\theta, \sigma)\right)\left|Z_{i}(\theta, \sigma)\right|
$$

and the cross-information quantity

$$
\mathcal{J}_{g_{1}}\left(f_{1}, g_{1}\right):=\int_{-\infty}^{+\infty} z^{2} \phi_{f_{1}}(z) \phi_{g_{1}}(z) g_{1}(z) d z
$$

we have the following result.

Proposition 3.4 Let $f_{1} \in \mathcal{F}_{1}$ and $g_{1} \in \mathcal{F}_{f_{1}}$. Then,

(i) $\hat{T}_{f_{1}}^{(n)}\left(\theta, \hat{\sigma}_{\#}\right)=\hat{T}_{f_{1}}^{(n)}(\theta, \sigma)+o_{\mathrm{P}}(1)$ is asymptotically normal, with mean zero under $\mathrm{P}_{\theta, \sigma, 0 ; g_{1}}^{(n)}$, mean $\tau \mathcal{J}_{g_{1}}\left(f_{1}, g_{1}\right) /\left[\mathcal{J}_{g_{1}}\left(f_{1}\right)\right]^{1 / 2}$ under $\mathrm{P}_{\theta, \sigma, n^{-1 / 2} \tau ; g_{1}}^{(n)}$, and variance one under both; 
(ii) the sequence of tests rejecting the null hypothesis $\mathcal{H}_{\theta}^{(n)}:=\bigcup_{g_{1} \in \mathcal{F}_{f_{1}}} \mathcal{H}_{\theta ; g_{1}}^{(n)}$ of symmetry with respect to specified $\theta$ whenever $\hat{T}_{f_{1}}^{(n)}\left(\theta, \hat{\sigma}_{\#}\right)$ exceeds the $(1-\alpha)$ standard normal quantile $z_{\alpha}$ is locally asymptotically most powerful, at asymptotic level $\alpha$, for $\mathcal{H}_{\theta}^{(n)}$ against $\bigcup_{\xi>0} \bigcup_{\sigma \in \mathbb{R}_{0}^{+}}\left\{\mathrm{P}_{\theta, \sigma, \xi ; f_{1}}^{(n)}\right\}$.

Such tests only make sense against fixed- $\theta$ alternatives. The fact that a test which is insensitive to perturbations (within $\mathcal{F}_{f_{1}}$ ) of the density $f_{1}$ is locally asymptotically optimal at $f_{1}$ indicates that semiparametric and parametric efficiency bounds coincide at $\xi=0$ and $f_{1}$. Note that the only reason for the validity of the test being restricted to densities $g_{1} \in \mathcal{F}_{f_{1}}$ is the applicability of Le Cam's third Lemma. The rank tests of Section 4 are valid under less restrictive conditions. Locally asymptotically maximin two-sided tests are easily derived along the same lines.

\subsection{Optimal parametric tests: unspecified density, unspecified location.}

When the location $\theta$ under the null hypothesis also is unspecified, additional care has to be taken about the asymptotic impact of estimating $\theta$ under unspecified density $g_{1}$. It follows from Le Cam's third Lemma that this impact on $\Delta_{f_{1} ; 3}^{(n)}$ can be obtained from the asymptotic behavior under $\mathrm{P}_{\theta, \sigma, 0 ; g_{1}}^{(n)}$ of

$$
\left(\begin{array}{c}
\Delta_{f_{1} ; 3}^{(n)}(\theta, \sigma, 0) \\
\Delta_{g_{1} ; 1}^{(n)}(\theta, \sigma, 0)
\end{array}\right)=n^{-1 / 2} \sum_{i=1}^{n}\left(\begin{array}{c}
-\phi_{f_{1}}\left(Z_{i}\right)\left|Z_{i}\right| \\
\frac{1}{\sigma} \phi_{g_{1}}\left(Z_{i}\right)
\end{array}\right),
$$

which is asymptotically normal with mean zero and asymptotic covariance matrix

$$
\left(\begin{array}{cc}
\mathcal{J}_{g_{1}}\left(f_{1}\right) & -\sigma^{-1} \mathcal{M}_{g_{1}}\left(f_{1}, g_{1}\right) \\
-\sigma^{-1} \mathcal{M}_{g_{1}}\left(f_{1}, g_{1}\right) & \sigma^{-2} \mathcal{I}\left(g_{1}\right)
\end{array}\right),
$$

where

$$
\mathcal{M}_{g_{1}}\left(f_{1}, g_{1}\right):=\int_{-\infty}^{+\infty}|z| \phi_{f_{1}}(z) \phi_{g_{1}}(z) g_{1}(z) d z
$$

Le Cam's third Lemma implies that replacing $\theta$ with a root- $n$ consistent $\hat{\theta}^{(n)}$ induces, under $\mathrm{P}_{\theta, \sigma, 0 ; g_{1}}^{(n)}$, a shift of the form

$$
\Delta_{f_{1} ; 3}^{(n)}\left(\hat{\theta}^{(n)}, \sigma, 0\right)-\Delta_{f_{1} ; 3}^{(n)}(\theta, \sigma, 0)=n^{1 / 2}\left(\hat{\theta}^{(n)}-\theta\right) \mathcal{M}_{g_{1}}\left(f_{1}, g_{1}\right) / \sigma,
$$

which is not $o_{\mathrm{P}}(1)$ as $n \rightarrow \infty$ (unless, of course, we are in the extremely particular case for which the underlying standardized density $g_{1}$ is such that $\left.\mathcal{M}_{g_{1}}\left(f_{1}, g_{1}\right)=0\right)$. In order to avoid this, let us modify $\Delta_{f_{1} ; 3}^{(n)}(\theta, \sigma, 0)$ into

$$
\Delta_{f_{1} ; 3}^{\eta ;(n)}(\theta, \sigma, 0):=-n^{-1 / 2} \sum_{i=1}^{n} \phi_{f_{1}}\left(Z_{i}\right)\left(\left|Z_{i}\right|-\eta\right)
$$

(compare with (3.7)). The joint distribution, under $\mathrm{P}_{\theta, \sigma, 0 ; g_{1}}^{(n)}$, of $\Delta_{f_{1} ; 3}^{\eta ;(n)}(\theta, \sigma, 0)$ and $\Delta_{g_{1} ; 1}^{(n)}(\theta, \sigma, 0)$ is still asymptotically normal with mean zero, but with asymptotic covariance $-\sigma^{-1}\left(\mathcal{M}_{g_{1}}\left(f_{1}, g_{1}\right)-\right.$ $\left.\eta \mathcal{I}_{g_{1}}\left(f_{1}, g_{1}\right)\right)$, where $\mathcal{I}_{g_{1}}\left(f_{1}, g_{1}\right):=\int_{-\infty}^{+\infty} \phi_{f_{1}}(z) \phi_{g_{1}}(z) g_{1}(z) d z$. Hence, letting $\eta=\eta_{g_{1}}\left(f_{1}, g_{1}\right):=$ $\mathcal{M}_{g_{1}}\left(f_{1}, g_{1}\right) / \mathcal{I}_{g_{1}}\left(f_{1}, g_{1}\right)$ cancels this covariance, and

$$
\Delta_{f_{1} ; 3}^{*(n)}(\theta, \sigma, 0):=\Delta_{f_{1} ; 3}^{\eta_{g_{1}}\left(f_{1}, g_{1}\right) ;(n)}(\theta, \sigma, 0)=n^{-1 / 2} \sum_{i=1}^{n} \phi_{f_{1}}\left(Z_{i}(\theta, \sigma)\right)\left(\eta_{g_{1}}\left(f_{1}, g_{1}\right)-\left|Z_{i}(\theta, \sigma)\right|\right)
$$


asymptotically is not affected, under $\mathrm{P}_{\theta, \sigma, 0 ; g_{1}}^{(n)}$, if $\hat{\theta}^{(n)}$ is substituted for $\theta$.

Note that $\eta_{g_{1}}\left(f_{1}, g_{1}\right)$, for $g_{1}=f_{1}$, coincides with $\eta\left(f_{1}\right)$, so that $\Delta_{f_{1} ; 3}^{*(n)}(\theta, \sigma, 0)$ under $\mathrm{P}_{\theta, \sigma, 0 ; f_{1}}^{(n)}$ coincides with the $f_{1}$-efficient central sequence for symmetry derived in (3.6). This $\Delta_{f_{1} ; 3}^{*(n)}$ thus has the desired property of being asymptotically equivalent to $\Delta_{f_{1}}^{*(n)}(\boldsymbol{\vartheta})$ under $\mathrm{P}_{\theta, \sigma, 0 ; f_{1}}^{(n)}$, and asymptotically uncorrelated with $\Delta_{g_{1} ; 1}^{(n)}$ and $\Delta_{g_{1} ; 2}^{(n)}$-hence, asymptotically insensitive (in probability) to root- $n$ perturbations of $\theta$ and $\sigma$ - under $\mathrm{P}_{\theta, \sigma, 0 ; g_{1}}^{(n)}$. The problem is that it still cannot be computed from the observations, since it explicitly depends on the unspecified $g_{1}$.

Assume therefore that $f_{1} \in \dot{\mathcal{F}}_{1}:=\left\{g_{1} \in \mathcal{F}_{1}: z \mapsto \phi_{g_{1}}(z)\right.$ admits a derivative $\left.\dot{\phi}_{g_{1}}\right\}$; put

$$
\begin{gathered}
\mathcal{I}^{(n) *}\left(\theta, \sigma ; f_{1}\right):=n^{-1} \sum_{i=1}^{n} \dot{\phi}_{f_{1}}\left(Z_{i}(\theta, \sigma)\right), \\
\mathcal{M}^{(n) *}\left(\theta, \sigma ; f_{1}\right):=n^{-1} \sum_{i=1}^{n} \operatorname{sign}\left(Z_{i}(\theta, \sigma)\right) \phi_{f_{1}}\left(Z_{i}(\theta, \sigma)\right)+n^{-1} \sum_{i=1}^{n}\left|Z_{i}(\theta, \sigma)\right| \dot{\phi}_{f_{1}}\left(Z_{i}(\theta, \sigma)\right),
\end{gathered}
$$

and

$$
\eta^{(n)}\left(\theta, \sigma ; f_{1}\right):=\mathcal{M}^{(n) *}\left(\theta, \sigma ; f_{1}\right) / \mathcal{I}^{(n) *}\left(\theta, \sigma ; f_{1}\right) .
$$

As for $g_{1}$, assume that it belongs to the subset $\mathcal{F}_{f_{1}}^{*}$ of $\dot{\mathcal{F}}_{1}$ for which

$$
\int_{-\infty}^{\infty} z^{k} \phi_{f_{1}}^{\ell}(z) \phi_{g_{1}}(z) g_{1}(z) d z<\infty, \quad k=0,1,2,3, \quad k=1,2
$$

and

$$
\int_{-\infty}^{\infty} z^{\ell} \dot{\phi}_{f_{1}}(z) \phi_{g_{1}}(z) g_{1}(z) d z<\infty, \quad \ell=0,1,2 .
$$

We then have the following result (as usual, we denote by $\hat{\theta}_{\#}$ and $\hat{\sigma}_{\#}$ any estimators satisfying $(\mathrm{C} 1)$ and $(\mathrm{C} 2)$ ); see the appendix for the proof.

Lemma 3.1 For $f_{1} \in \mathcal{F}_{1}^{*}$ and $g_{1} \in \mathcal{F}_{f_{1}}^{*}$,

$$
\Delta_{f_{1} ; 3}^{*(n)}\left(\eta^{(n)}\left(\hat{\theta}_{\#}, \hat{\sigma}_{\#} ; f_{1}\right), \hat{\theta}_{\#}, \hat{\sigma}_{\#}\right)-\Delta_{f_{1} ; 3}^{*(n)}\left(\eta_{g_{1}}\left(f_{1}, g_{1}\right), \theta, \sigma\right)=o_{\mathrm{P}}(1)
$$

as $n \rightarrow \infty$, under $\mathrm{P}_{\theta, \sigma, 0 ; g_{1}}^{(n)}$.

Summing up, letting, for $f_{1} \in \mathcal{F}_{1}^{*}$,

$$
\hat{T}_{f_{1}}^{*(n)}(\theta, \sigma):=\frac{1}{\sqrt{n \gamma^{(n) *}\left(\theta, \sigma ; f_{1}\right)}} \sum_{i=1}^{n} \phi_{f_{1}}\left(Z_{i}(\theta, \sigma)\right)\left(\eta^{(n)}\left(\theta, \sigma ; f_{1}\right)-\left|Z_{i}(\theta, \sigma)\right|\right)
$$

with

$$
\begin{aligned}
& \gamma^{(n) *}\left(\theta, \sigma ; f_{1}\right):=\mathcal{J}^{(n)}\left(\theta, \sigma ; f_{1}\right)-2 \eta^{(n)}\left(\theta, \sigma ; f_{1}\right) \mathcal{M}^{(n)}\left(\theta, \sigma ; f_{1}\right)+\left(\eta^{(n)}\left(\theta, \sigma ; f_{1}\right)\right)^{2} \mathcal{I}^{(n)}\left(\theta, \sigma ; f_{1}\right), \\
& \mathcal{I}^{(n)}\left(\theta, \sigma ; f_{1}\right):=n^{-1} \sum_{i=1}^{n} \phi_{f_{1}}^{2}\left(Z_{i}(\theta, \sigma)\right) \quad \text { and } \quad \mathcal{M}^{(n)}\left(\theta, \sigma ; f_{1}\right):=n^{-1} \sum_{i=1}^{n}\left|Z_{i}(\theta, \sigma)\right| \phi_{f_{1}}^{2}\left(Z_{i}(\theta, \sigma)\right),
\end{aligned}
$$

we have shown the following result. 
Proposition 3.5 For any $f_{1} \in \mathcal{F}_{1}^{*}$ and $g_{1} \in \mathcal{F}_{f_{1}}^{*}$,

(i) $\hat{T}_{f_{1}}^{*(n)}\left(\hat{\theta}_{\#}, \hat{\sigma}_{\#}\right)=\hat{T}_{f_{1}}^{*(n)}(\theta, \sigma)+o_{\mathrm{P}}(1)$ is asymptotically normal, with mean zero under $\mathrm{P}_{\theta, \sigma, 0: g_{1}}^{(n)}$, mean

$$
\tau \frac{\mathcal{J}_{g_{1}}\left(f_{1}, g_{1}\right)-\eta_{g_{1}}\left(f_{1}, g_{1}\right) \mathcal{M}_{g_{1}}\left(f_{1}, g_{1}\right)}{\left[\eta_{g_{1}}^{2}\left(f_{1}, g_{1}\right) \mathcal{I}_{g_{1}}\left(f_{1}\right)-2 \eta_{g_{1}}\left(f_{1}, g_{1}\right) \mathcal{M}_{g_{1}}\left(f_{1}\right)+\mathcal{J}_{g_{1}}\left(f_{1}\right)\right]^{1 / 2}}
$$

under $\mathrm{P}_{\theta, \sigma, n^{-1 / 2} \tau ; g_{1}}^{(n)}$, and variance one under both;

(ii) the sequence of tests rejecting the null hypothesis $\mathcal{H}^{(n)}:=\bigcup_{g_{1} \in \mathcal{F}_{f_{1}}^{*}} \bigcup_{\theta \in \mathbb{R}} \mathcal{H}_{\theta ; g_{1}}^{(n)}$ of symmetry (with unspecified location $\theta$ and unspecified standardized density $\left.g_{1}\right)$ whenever $T_{f_{1}}^{(n) *}\left(\hat{\theta}_{\#}, \hat{\sigma}_{\#}\right)$ exceeds the $(1-\alpha)$ standard normal quantile $z_{\alpha}$ is locally asymptotically optimal (most stringent), at asymptotic level $\alpha$, for $\mathcal{H}^{(n)}$ against $\bigcup_{\xi>0} \bigcup_{\theta \in \mathbb{R}} \bigcup_{\sigma \in \mathbb{R}_{0}^{+}}\left\{\mathrm{P}_{\theta, \sigma, \xi: f_{1}}^{(n)}\right\}$.

The assumption that $f_{1} \in \mathcal{F}_{1}^{*}$ places a restriction on the score function which, mild as it may be, nevertheless excludes the classical double-exponential (sign test) scores, since $\phi_{f_{\mathcal{L}}}(z)=$ $\operatorname{sign}(z) / d$ is not differentiable. In this case, however, $\mathcal{I}_{g_{1}}\left(f_{\mathcal{L}}, g_{1}\right)$ reduces to $2 g_{1}(0) / d$-which is consistently estimated by $\mathcal{I}^{(n) \odot}\left(f_{\mathcal{L}}\right):=2 \hat{g}_{1}(0) / d$ (where $\hat{g}_{1}$ is any consistent kernel estimator of $\left.g_{1}\right)$ - and $\mathcal{M}_{g_{1}}\left(f_{\mathcal{L}}, g_{1}\right)$ reduces to $\frac{1}{d}$. Then, $\eta^{(n)}\left(\hat{\theta}, \hat{\sigma} ; f_{\mathcal{L}}\right):=1 /\left(2 \hat{g}_{1}(0)\right)$ is such that $\eta^{(n)}\left(\hat{\theta}, \hat{\sigma} ; f_{\mathcal{L}}\right)-$ $\eta_{g_{1}}\left(f_{\mathcal{L}}, g_{1}\right)=o_{\mathrm{P}}(1)$ under $\mathrm{P}_{\theta, \sigma, 0 ; g_{1}}^{(n)}$, and the conclusions of Proposition 3.5 still hold.

\subsection{Pseudo-Gaussian tests.}

For $f_{1}=\phi_{1}$ and $g_{1} \in \mathcal{F}_{\phi_{1}}=\mathcal{F}_{\phi_{1}}^{*}$, Propositions 3.4 and 3.5 yield pseudo-Gaussian tests, that is, tests that are valid under a broad class of non-Gaussian densities, while remaining optimal under Gaussian ones. Defining $m_{k}^{(n)}(\theta):=n^{-1} \sum_{i=1}^{n}\left(X_{i}-\theta\right)^{k}$ and $m_{k}^{*(n)}(\theta):=n^{-1} \sum_{i=1}^{n}\left|X_{i}-\theta\right|^{k}$, the test statistic (3.10) reduces to

$$
\hat{T}_{\phi_{1}}^{(n)}(\theta):=\frac{-1}{\sqrt{n m_{4}^{(n)}(\theta)}} \sum_{i=1}^{n} \operatorname{sign}\left(X_{i}-\theta\right)\left(X_{i}-\theta\right)^{2} .
$$

This pseudo-Gaussian test has the advantage, when compared with the classical procedure based on $m_{3}^{(n)}(\theta)$, that it only requires finite fourth-order moments instead of the moments of order six needed for the asymptotic normality of the empirically standardized form (1.1) of $m_{3}^{(n)}(\theta)$. Define $\mu_{k}:=\int_{-\infty}^{\infty} z^{k} g_{1}(z) d z$. We then have the following special case of Proposition 3.4.

Proposition 3.6 Let $g_{1} \in \mathcal{F}_{\phi_{1}}$. Then,

(i) $\hat{T}_{\phi_{1}}^{(n)}(\theta)$ is asymptotically normal, with mean zero under $\mathrm{P}_{\theta, \sigma, 0 ; g_{1}}^{(n)}$, mean $3 \tau \mu_{2} /\left[\mu_{4}\right]^{1 / 2}$ under $\mathrm{P}_{\theta, \sigma, n^{-1 / 2} \tau ; g_{1}}^{(n)}$, and variance one under both.

(ii) The sequence of tests rejecting the null hypothesis $\mathcal{H}_{\theta}^{(n)}:=\bigcup_{g_{1} \in \mathcal{F}_{\phi_{1}}} \mathcal{H}_{\theta ; g_{1}}^{(n)}$ of symmetry with respect to specified $\theta$ whenever $\hat{T}_{\phi_{1}}^{(n)}(\theta)$ exceeds the $(1-\alpha)$ standard normal quantile $z_{\alpha}$ is locally asymptotically most powerful, at asymptotic level $\alpha$, for $\mathcal{H}_{\theta}^{(n)}$ against $\bigcup_{\xi>0} \bigcup_{\sigma \in \mathbb{R}_{0}^{+}}\left\{\mathrm{P}_{\theta, \sigma, \xi ; \phi_{1}}^{(n)}\right\}$. 
In the unspecified location case, the test statistic (3.14) reduces to

$$
\hat{T}_{\phi_{1}}^{*(n)}(\theta)=\frac{\sum_{i=1}^{n}\left(X_{i}-\theta\right)\left(2 m_{1}^{*(n)}(\theta)-\left|X_{i}-\theta\right|\right)}{\sqrt{n\left(m_{4}^{(n)}(\theta)-4 m_{1}^{*(n)}(\theta) m_{3}^{*(n)}(\theta)+4\left(m_{1}^{*(n)}(\theta)\right)^{2} m_{2}^{(n)}(\theta)\right)}}
$$

If one uses the sample mean for the estimation of $\theta$, the first term in the numerator of (3.15) disappears. Note that the assumption that $g_{1} \in\left(\mathcal{F}_{\phi_{1}}^{*}=\right) \mathcal{F}_{\phi_{1}}$ implies that $g_{1}$ has finite moments of order four. Letting $\mu_{k}^{*}:=\int_{-\infty}^{\infty}|z|^{k} g_{1}(z) d z$, we thus have the following result.

Proposition 3.7 Let $g_{1} \in \mathcal{F}_{\phi_{1}}$. Then,

(i) $\hat{T}_{\phi_{1}}^{*(n)}\left(\hat{\theta}_{\#}\right)=\hat{T}_{\phi_{1}}^{*(n)}(\theta)+o_{\mathrm{P}}(1)$ is asymptotically normal, with mean zero under $\mathrm{P}_{\theta, \sigma, 0: g_{1}}^{(n)}$, mean $\tau\left(3 \mu_{2}-4 \mu_{1}^{* 2}\right)\left[4 \mu_{1}^{* 2} \mu_{2}-4 \mu_{1}^{*} \mu_{3}^{*}+\mu_{4}\right]^{-1 / 2}$ under $\mathrm{P}_{\theta, \sigma, n^{-1 / 2} \tau ; g_{1}}^{(n)}$, and variance one under both.

(ii) The sequence of tests rejecting the null hypothesis $\mathcal{H}^{(n)}:=\bigcup_{g_{1} \in \mathcal{F}_{\phi_{1}}} \bigcup_{\theta \in \mathbb{R}} \mathcal{H}_{\theta ; g_{1}}^{(n)}$ of symmetry (with unspecified location $\theta$ and unspecified standardized density $\left.g_{1}\right)$ whenever $T_{f_{1}}^{(n) *}\left(\hat{\theta}_{\#}, \hat{\sigma}_{\#}\right)$ exceeds the $(1-\alpha)$ standard normal quantile $z_{\alpha}$ is locally asymptotically optimal (most stringent), at asymptotic level $\alpha$, for $\mathcal{H}^{(n)}$ against $\bigcup_{\xi>0} \bigcup_{\theta \in \mathbb{R}} \bigcup_{\sigma \in \mathbb{R}_{0}^{+}}\left\{\mathrm{P}_{\theta, \sigma, \xi ; \phi_{1}}^{(n)}\right\}$.

For the sake of completeness, we also provide (with the same notation) the following result on the asymptotic behavior of the (suboptimal) tests based on $m_{3}^{(n)}(\theta)$ and $b_{1}^{(n)}$. The proof is straightforward, and details are left to the reader.

Proposition 3.8 Let $g_{1} \in \mathcal{F}_{\phi_{1}}$. Then,

(i) $n^{1 / 2} m_{3}^{(n)}(\theta) /\left(m_{6}^{(n)}(\theta)\right)^{1 / 2}$ is asymptotically normal, with mean zero under $\mathrm{P}_{\theta, \sigma, 0: g_{1}}^{(n)}$, mean $\tau\left[4 \mu_{3}^{*}\right] / \mu_{6}^{1 / 2}$ under $\mathrm{P}_{\theta, \sigma, n^{-1 / 2} \tau ; g_{1}}^{(n)}$, and variance one under both.

(ii) $n^{1 / 2} m_{3}^{(n)} /\left(m_{6}^{(n)}-6 s_{n}^{2} m_{4}^{(n)}+9 s_{n}^{6}\right)^{1 / 2}$ is asymptotically normal, with mean zero under $\mathrm{P}_{\theta, \sigma, 0: g_{1}}^{(n)}$, mean $\tau\left[-4 \mu_{3}^{*}+6 \mu_{1}^{*} \mu_{2}\right] /\left[\mu_{6}-6 \mu_{2} \mu_{4}+9 \mu_{2}^{3}\right]^{1 / 2}$ under $\mathrm{P}_{\theta, \sigma, n^{-1 / 2} \tau ; g_{1}}^{(n)}$, and variance one under both.

It should be stressed, however, that none of those classical tests are (locally and asymptotically) optimal in any Fechner family.

\section{Rank-based tests for symmetry.}

\subsection{Signed-rank versions of the central sequence.}

As mentioned in the introduction, the hypothesis of symmetry enjoys strong group invariance features. The null hypothesis $\mathcal{H}_{\theta}^{(n)}$ of symmetry with respect to $\theta$ indeed is generated by the group $\mathcal{G}_{\theta}^{(n)}$,。of all transformations $\mathcal{G}_{h}$ of $\mathbb{R}^{n}$ such that $\mathcal{G}_{h}\left(x_{1}, \ldots, x_{n}\right):=\left(h\left(x_{1}\right), \ldots h\left(x_{n}\right)\right)$, where $\lim _{x \rightarrow \pm \infty} h(x)= \pm \infty$, and $x \mapsto h(x)$ is continuous, monotone increasing, and skew-symmetric with respect to $\theta$ (i.e. $h(\theta-z)=-h(\theta+z)$ ). A maximal invariant for that group is known to be the vector of signs $\left(s_{1}(\theta), \ldots, s_{n}(\theta)\right)$, along with the vector of $\operatorname{ranks}\left(R_{+, 1}^{(n)}(\theta), \ldots, R_{+, n}^{(n)}(\theta)\right)$, where $s_{i}(\theta)$ is the sign of $X_{i}-\theta$ and $R_{+, i}^{(n)}(\theta)$ the rank of $\left|X_{i}-\theta\right|$ among $\left|X_{1}-\theta\right|, \ldots,\left|X_{n}-\theta\right|$. 
General results on semiparametric efficiency (Hallin and Werker 2003) indicate that, in such context, the expectation of the central sequence $\boldsymbol{\Delta}_{f_{1}}^{(n)}(\boldsymbol{\vartheta})$ conditional on those signs and ranks yields a version of the semiparametrically efficient (at $f_{1}$ and $\boldsymbol{\vartheta}$ ) central sequence. The only component of the central sequence $\Delta_{f_{1}}^{(n)}$ which is used in this section is the $\xi$-component $\Delta_{f_{1} ; 3}^{(n)}$, with signed-rank version

$$
{\underset{\sim}{f_{1} ; 3}}_{(n)}^{(n)}(\theta):=-n^{-1 / 2} \sum_{i=1}^{n} s_{i}(\theta) \phi_{f_{1}}\left(F_{1+}^{-1}\left(\frac{R_{+, i}^{(n)}(\theta)}{n+1}\right)\right) F_{1+}^{-1}\left(\frac{R_{+, i}^{(n)}(\theta)}{n+1}\right),
$$

where $F_{1+}=2 F_{1}-1$ and $F_{1}$ denote the distribution functions of $\left|Z_{i}\right|$ and $Z_{i}$, respectively, when $Z_{i}$ has density $f_{1}$. Later on, however, we also will need the rank-based version

$$
\underset{\sim}{\Delta} f_{1 ; 1}^{(n)}(\theta, \sigma):=\frac{n^{-1 / 2}}{\sigma} \sum_{i=1}^{n} s_{i}(\theta) \phi_{f_{1}}\left(F_{1+}^{-1}\left(\frac{R_{+, i}^{(n)}(\theta)}{n+1}\right)\right)
$$

of the $\theta$-component.

Denote by $\mathcal{F}_{1}^{\square}$ the collection of standardized densities $f_{1} \in \mathcal{F}_{1}$ for which $\phi_{f_{1}}$ can be written as the difference of two monotone increasing functions. The following asymptotic representation result then follows from the classical Hájek theory for linear signed-rank statistics (see, e.g., Chapter 3 of Puri and Sen 1985).

Proposition 4.1 Let $f_{1} \in \mathcal{F}_{1}^{\square}$ and $g_{1} \in \mathcal{F}_{0}$, with distribution functions $F_{1}$ and $G_{1}$, respectively. Then,

(i) $\underset{\sim}{\Delta}(n) ; 3(\theta)=\mathrm{E}\left[\Delta_{f_{1} ; 3}^{(n)}(\theta, \sigma, 0) \mid s_{1}(\theta), \ldots, s_{n}(\theta), R_{+, 1}^{(n)}(\theta), \ldots, R_{+, n}^{(n)}(\theta)\right]+o_{L^{2}}(1)$

$$
=-n^{-1 / 2} \sum_{i=1}^{n} \phi_{f_{1}}\left(F_{1}^{-1}\left(G_{1}\left(Z_{i}^{(n)}(\theta, \sigma)\right)\right)\right)\left|F_{1}^{-1}\left(G_{1}\left(Z_{i}^{(n)}(\theta, \sigma)\right)\right)\right|+o_{L^{2}}(1)
$$

as $n \rightarrow \infty$, under $\mathrm{P}_{\theta, \sigma, 0 ; g_{1}}^{(n)}$, and hence $\underset{\sim}{\Delta}(n) ;(\theta)=\Delta_{f_{1} ; 3}^{(n)}(\theta, \sigma, 0)+o \mathrm{P}(1)$ under $\mathrm{P}_{\theta, \sigma, 0 ; f_{1}}^{(n)}$;

(ii) $\underset{\sim}{\Delta}(n) ; 3$ has mean zero and variance

$$
\mathcal{J}^{(n)}\left(f_{1}\right):=\frac{1}{n} \sum_{r=1}^{n} \phi_{f_{1}}^{2}\left(F_{1+}^{-1}\left(\frac{r}{n+1}\right)\right)\left(F_{1+}^{-1}\left(\frac{r}{n+1}\right)\right)^{2}
$$

under $\mathrm{P}_{\theta, \sigma, 0 ; g_{1}}^{(n)}$. Moreover, $\mathcal{J}^{(n)}\left(f_{1}\right)=\mathcal{J}\left(f_{1}\right)+o(1)$ as $n \rightarrow \infty$.

The asymptotic equivalence under $\mathrm{P}_{\theta, \sigma, 0 ; f_{1}}^{(n)}$ of $\underset{\sim}{\Delta} f_{1} ; 3(\theta)$ and $\Delta_{f_{1} ; 3}^{(n)}(\theta, \sigma, 0)$ confirms the remark made after Proposition 3.4 that semiparametric and parametric efficiency bounds for $\xi$ in this problem coincide.

\subsection{Optimal signed-rank tests of symmetry: specified location.}

Proposition 4.1 immediately allows for constructing a distribution-free rank-based test of symmetry with respect to a specified location $\theta$. Let

$$
\underset{\sim}{T} f_{1}^{(n)}(\theta):=\frac{{\stackrel{\sim}{\sim} f_{1} ; 3}_{(n)}^{(n)}}{\sqrt{\mathcal{J}^{(n)}\left(f_{1}\right)}}=\frac{-1}{\sqrt{n \mathcal{J}^{(n)}\left(f_{1}\right)}} \sum_{i=1}^{n} s_{i}(\theta) \phi_{f_{1}}\left(F_{1+}^{-1}\left(\frac{R_{+, i}^{(n)}(\theta)}{n+1}\right)\right)\left|F_{1+}^{-1}\left(\frac{R_{+, i}^{(n)}(\theta)}{n+1}\right)\right|,
$$


define the cross-information coefficient

$$
\mathcal{L}\left(f_{1}, g_{1}\right):=\int_{0}^{1}\left|F_{1}^{-1}(u)\right|\left|G_{1}^{-1}(u)\right| \phi_{f_{1}}\left(F_{1}^{-1}(u)\right) \phi_{g_{1}}\left(G_{1}^{-1}(u)\right) d u
$$

and denote by $\underset{\sim}{\mathcal{F}} f_{1}:=\left\{g_{1} \in \mathcal{F}_{1} \mid \mathcal{L}\left(f_{1}, g_{1}\right)<\infty\right\}$ the class of densities for which this integral exists.

Proposition 4.2 Let $f_{1} \in \mathcal{F}_{1}^{\square}$. Then,

(i) $\underset{\sim}{T} f_{1}^{(n)}(\theta)$ is asymptotically normal, with mean zero under $\mathrm{P}_{\theta, \sigma, 0 ; g_{1}}^{(n)}, g_{1} \in \mathcal{F}_{0}$, mean $\tau \mathcal{L}\left(f_{1}, g_{1}\right) / \sqrt{\mathcal{J}\left(f_{1}\right)}$ under $\mathrm{P}_{\theta, \sigma, n^{-1 / 2} \tau ; g_{1}}^{(n)}, g_{1} \in \underset{\sim}{\mathcal{F}} f_{1}$, and variance one under both;

(ii) the sequence of tests rejecting the null hypothesis $\mathcal{H}_{\theta}^{(n)}:=\bigcup_{g_{1} \in \mathcal{F}_{0}} \bigcup_{\sigma \in \mathbb{R}_{0}^{+}} \mathrm{P}_{\theta, \sigma, 0 ; g_{1}}^{(n)}$ of symmetry with respect to $\theta$ whenever $\underset{\sim}{T} f_{1}^{(n)}(\theta)$ exceeds the $(1-\alpha)$ standard normal quantile $z_{\alpha}$ is locally asymptotically most powerful, at asymptotic level $\alpha$, for $\mathcal{H}_{\theta}^{(n)}$ against $\bigcup_{\xi>0} \bigcup_{\sigma \in \mathbb{R}_{0}^{+}}\left\{\mathrm{P}_{\theta, \sigma, \xi: f_{1}}^{(n)}\right\}$.

Only asymptotic critical values are reported in Part (ii) of the proposition, but exact ones of course also can be considered (or simulated), as the test is entirely distribution-free. The two-sided version also readily follows. Here again, these tests should be used against fixed- $\theta$ alternatives only.

\subsection{Optimal signed-rank tests of symmetry: unspecified location.}

When $\theta$ is unspecified under the null, a consistent estimator $\hat{\theta}$ has to be substituted for $\theta$, yielding aligned signs $s_{i}(\hat{\theta})$ and $\operatorname{ranks} R_{+, i}^{(n)}(\hat{\theta})$. The effect of this alignment procedure is taken care of in a similar way as in Section 3.5. The asymptotic joint distribution, under $\mathrm{P}_{\theta, \sigma, 0 ; g_{1}}^{(n)}$, of

$$
\left(\begin{array}{c}
\underset{\sim}{\Delta_{f_{1} ; 3}^{(n)}(\theta)} \\
\Delta_{g_{1} ; 1}^{(n)}(\theta, \sigma, 0)
\end{array}\right)=n^{-1 / 2} \sum_{i=1}^{n}\left(\begin{array}{c}
-\phi_{f_{1}}\left(F_{1}^{-1}\left(G_{1}\left(Z_{i}^{(n)}(\theta, \sigma)\right)\right)\right)\left|F_{1}^{-1}\left(G_{1}\left(Z_{i}^{(n)}(\theta, \sigma)\right)\right)\right| \\
\frac{1}{\sigma} \phi_{g_{1}}\left(Z_{i}\right)
\end{array}\right)+o_{\mathrm{P}}(1)
$$

(in view of the asymptotic representation of Part (i) of Proposition 4.1) is asymptotically normal with mean zero and asymptotic covariance matrix

$$
\left(\begin{array}{cc}
\mathcal{J}\left(f_{1}\right) & -\sigma^{-1} \mathcal{M}\left(f_{1}, g_{1}\right) \\
-\sigma^{-1} \mathcal{M}\left(f_{1}, g_{1}\right) & \sigma^{-2} \mathcal{I}\left(g_{1}\right)
\end{array}\right)
$$

where we let

$$
\begin{gathered}
\mathcal{M}\left(f_{1}, g_{1}\right):=\int_{0}^{1}\left|F_{1}^{-1}(u)\right| \phi_{f_{1}}\left(F_{1}^{-1}(u)\right) \phi_{g_{1}}\left(G_{1}^{-1}(u)\right) d u . \\
\mathcal{I}\left(f_{1}, g_{1}\right):=\int_{0}^{1} \phi_{f_{1}}\left(F_{1}^{-1}(u)\right) \phi_{g_{1}}\left(G_{1}^{-1}(u)\right) d u
\end{gathered}
$$

and denote by $\underset{\sim}{\mathcal{F}} f_{1}:=\left\{g_{1} \in \mathcal{F}_{1} \mid \mathcal{I}\left(f_{1}, g_{1}\right)<\infty, \mathcal{L}\left(f_{1}, g_{1}\right)<\infty\right.$, and $\left.\mathcal{M}\left(f_{1}, g_{1}\right)<\infty\right\}$

Letting $\eta\left(f_{1}, g_{1}\right):=\mathcal{M}\left(f_{1}, g_{1}\right) / \mathcal{I}\left(f_{1}, g_{1}\right)$, Le Cam's third Lemma implies that 


$$
\underset{\sim}{\Delta} f_{1}^{(n)}\left(\eta\left(f_{1}, g_{1}\right) ; \theta\right):=n^{-1 / 2} \sum_{i=1}^{n} s_{i}(\theta) \phi_{f_{1}}\left(F_{1+}^{-1}\left(\frac{R_{+, i}^{(n)}(\theta)}{n+1}\right)\right)\left(\eta\left(f_{1}, g_{1}\right)-F_{1+}^{-1}\left(\frac{R_{+, i}^{(n)}(\theta)}{n+1}\right)\right),
$$

hence also $\underset{\sim}{\Delta} f_{1}^{(n)}\left(\eta^{(n)}\left(f_{1} ; \theta\right) ; \theta\right)$ where $\eta^{(n)}\left(f_{1} ; \theta\right)$ is any consistent (under $\mathrm{P}_{\theta, \sigma, 0 ; g_{1}}^{(n)}$ ) estimator of $\eta\left(f_{1}, g_{1}\right)$, are asymptotically insensitive to root- $n$ perturbations of $\theta$. The same reasoning as in Section 3.5 yields the asymptotic equivalence

$$
\underset{\sim}{\Delta} f_{1}^{(n)}\left(\eta^{(n)}\left(f_{1} ; \hat{\theta}_{\#}\right) ; \hat{\theta}_{\#}\right)-{\underset{\sim}{f_{1}}}_{f_{1}}^{(n)}\left(\underline{\eta}^{(n)}\left(f_{1} ; \theta\right) ; \theta\right)=o_{\mathrm{P}}(1) \quad \text { under } \mathrm{P}_{\theta, \sigma, 0 ; g_{1}}^{(n)}
$$

for any estimator $\hat{\theta}_{\#}$ of $\theta$ satisfying $(\mathrm{C} 1)$ and (C2) and any

$$
g_{1} \in \mathcal{F}_{\sim}^{*} f_{1}:=\left\{g_{1} \in \mathcal{F}_{0} \mid \mathcal{I}\left(g_{1}\right)<\infty, \mathcal{I}\left(f_{1}, g_{1}\right)<\infty \text {, and } \mathcal{M}\left(f_{1}, g_{1}\right)<\infty\right\}
$$

Estimating $\underline{\eta}\left(f_{1}, g_{1}\right)$ consistently, however, is more delicate, and a precise description of $\eta^{(n)}\left(f_{1} ; \theta\right)$ is postponed to Section 4.5. Note however that $\eta\left(f_{1}, f_{1}\right)=\eta\left(f_{1}\right)$.

Defining

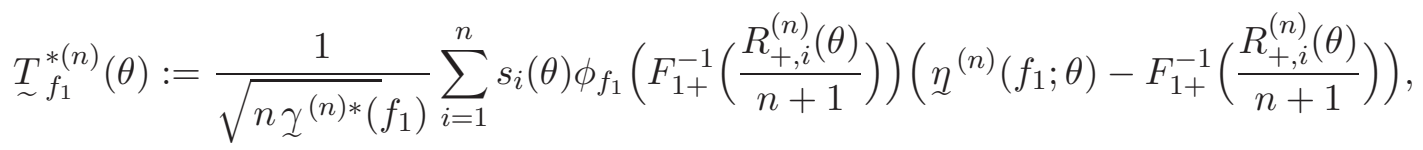

where

$$
\underline{\chi}^{*(n)}\left(f_{1}\right):=\frac{1}{n} \sum_{r=1}^{n} \phi_{f_{1}}^{2}\left(F_{1+}^{-1}\left(\frac{r}{n+1}\right)\right)\left(\underline{\eta}^{(n)}\left(f_{1} ; \theta\right)-F_{1+}^{-1}\left(\frac{r}{n+1}\right)\right)^{2},
$$

we have established the following result.

Proposition 4.3 Let $f_{1} \in \mathcal{F}_{1}^{\square}$. Then,

(i) $\underset{\sim}{T} f_{1}^{*(n)}\left(\hat{\theta}_{\#}\right)$ is asymptotically normal, with mean zero under $\mathrm{P}_{\theta, \sigma, 0 ; g_{1}}^{(n)}, g_{1} \in \underset{\sim}{\mathcal{F}_{f_{1}}^{*}}$, mean

$$
\tau \frac{\mathcal{L}\left(f_{1}, g_{1}\right)-\mathcal{M}\left(g_{1}, f_{1}\right) \underline{\eta}\left(f_{1}, g_{1}\right)}{\left[\mathcal{J}\left(f_{1}\right)-2 \eta\left(f_{1}, g_{1}\right) \mathcal{M}\left(f_{1}\right)+\underline{\eta}^{2}\left(f_{1}, g_{1}\right) \mathcal{I}\left(f_{1}\right)\right]^{1 / 2}}
$$

under $\mathrm{P}_{\theta, \sigma, n^{-1 / 2} \tau ; g_{1}}^{(n)}, g_{1} \in \underset{\sim}{\mathcal{F}} f_{1}$, and variance one under both;

(ii) the sequence of tests rejecting the null hypothesis $\mathcal{H}^{(n)}:=\bigcup_{g_{1} \in \underset{\sim}{\mathcal{F}}{ }_{f_{1}}^{*}} \bigcup_{\theta \in \mathbb{R}} \bigcup_{\sigma \in \mathbb{R}_{0}^{+}} \mathrm{P}_{\theta, \sigma, 0 ; g_{1}}^{(n)}$

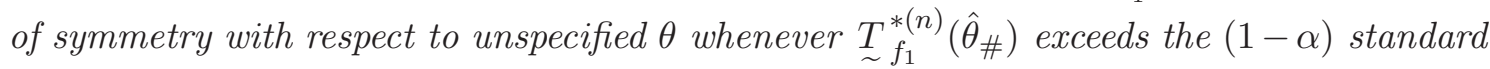
normal quantile $z_{\alpha}$ is locally asymptotically optimal (most stringent), at asymptotic level $\alpha$, for $\mathcal{H}^{(n)}$ against $\bigcup_{\xi>0} \bigcup_{\theta \in \mathbb{R}} \bigcup_{\sigma \in \mathbb{R}_{0}^{+}}\left\{\mathrm{P}_{\theta, \sigma, \xi: f_{1}}^{(n)}\right\}$. 


\subsection{Wilcoxon, sign, and normal score tests of symmetry.}

The statistics (4.2) and (4.3) are providing general forms for the optimal signed-rank tests of symmetry, under specified and unspecified location, respectively. Important particular cases are the sign, Wilcoxon and van der Waerden (normal score) tests statistics, which are optimal at double exponential, logistic, and normal distributions, respectively.

The normal score tests use $f_{1}=\phi_{1}$. One easily verifies that $F_{1+}^{-1}(u)=a^{-1 / 2} \Phi^{-1}\left(\frac{u+1}{2}\right)$ and $\phi_{f_{1}}\left(F_{1+}^{-1}(u)\right)=a^{1 / 2} \Phi^{-1}\left(\frac{u+1}{2}\right)$, where $\Phi$ denotes the standard normal distribution function and $a$ is defined as in Section 2. When the location $\theta$ is specified, the statistic (4.2) reduces to

$$
\underset{\sim}{T} \mathrm{vdW}^{(n)}(\theta):=\underset{\sim}{T} \phi_{1}^{(n)}(\theta):=\frac{-1}{\sqrt{n \mathcal{J}^{(n)}\left(\phi_{1}\right)}} \sum_{i=1}^{n} s_{i}(\theta) \Phi^{-1}\left(\frac{n+1+R_{+, i}^{(n)}(\theta)}{2(n+1)}\right) \Phi^{-1}\left(\frac{n+1+R_{+, i}^{(n)}(\theta)}{2(n+1)}\right),
$$

where $\mathcal{J}^{(n)}\left(\phi_{1}\right):=n^{-1} \sum_{r=1}^{n}\left(\Phi^{-1}\left(\frac{n+1+r}{2(n+1)}\right)\right)^{4}$. When the location $\theta$ under the null is unspecified, the normal score test statistic can be written as

$$
\underset{\sim}{T_{\operatorname{vdW}}^{*(n)}}(\hat{\theta}):=\frac{1}{\sqrt{n \mathcal{Y}^{*(n)}\left(\phi_{1}\right)}} \sum_{i=1}^{n} s_{i}(\theta) \Phi^{-1}\left(\frac{n+1+R_{+, i}^{(n)}(\hat{\theta})}{2(n+1)}\right)\left(\underline{\eta}^{(n)}\left(\phi_{1} ; \theta\right)-\Phi^{-1}\left(\frac{n+1+R_{+, i}^{(n)}(\hat{\theta})}{2(n+1)}\right)\right),
$$

where $\mathcal{Z}^{*(n)}\left(\phi_{1}\right):=n^{-1} \sum_{r=1}^{n}\left(\Phi^{-1}\left(\frac{n+1+r}{2(n+1)}\right)\right)^{2}\left(\underline{\eta}^{(n)}\left(\phi_{1} ; \theta\right)-\Phi^{-1}\left(\frac{n+1+r}{2(n+1)}\right)\right)^{2}$.

In the Wilcoxon case, $f_{1}$ is a standardized logistic. One easily checks, in that case, that $F_{1+}^{-1}(u)=b^{-1 / 2} \ln \frac{1+u}{1-u}$ and $\phi_{f_{1}}\left(F_{1+}^{-1}(u)\right)=b^{-1 / 2} u$ (with $b$ defined in Section 2). Therefore, (4.2) reduces to

$$
\underset{\sim}{T}(n)(\theta):=\frac{-1}{\sqrt{n \mathcal{J}^{(n)}\left(f_{L o g}\right)}} \sum_{i=1}^{n} s_{i}(\theta) \frac{R_{+, i}^{(n)}(\theta)}{n+1} \ln \frac{n+1+R_{+, i}^{(n)}(\theta)}{n+1-R_{+, i}^{(n)}(\theta)},
$$

where $\mathcal{J}^{(n)}\left(f_{\text {Log }}\right):=n^{-1} \sum_{r=1}^{n}\left(\frac{r}{n+1}\right)^{2}\left(\ln \frac{n+1+r}{n+1-r}\right)^{2}$. When the location under the null is unspecified, the Wilcoxon version

$$
\underset{\sim}{T} \log ^{*(n)}\left(\hat{\theta}_{\#}\right):=\frac{1}{\sqrt{n \chi^{*(n)}\left(f_{L o g}\right)}} \sum_{i=1}^{n} s_{i}(\theta) \frac{R_{+, i}^{(n)}(\theta)}{n+1}\left(\underline{\eta}^{(n)}\left(f_{L o g} ; \theta\right)-\left|\ln \frac{n+1+R_{+, i}^{(n)}(\theta)}{n+1-R_{+, i}^{(n)}(\theta)}\right|\right)
$$

of (4.3) is easily derived once the estimator $\eta^{(n)}\left(f_{\text {Log }} ; \theta\right)$ is appropriately defined.

The sign tests for symmetry use $f_{1}=f_{\mathcal{L}}$. One can easily check that $F_{1+}^{-1}(u)=-d \ln (1-u)$ and $\phi_{f_{1}}\left(F_{1+}^{-1}(u)\right)=1 / d$ (with $d$ defined in Section 2). Therefore, these sign tests take the form

$$
\underset{\sim}{T} f_{\mathcal{L}}^{(n)}(\theta):=\frac{-1}{\sqrt{n \mathcal{J}_{\sim}^{(n)}\left(f_{\mathcal{L}}\right)}} \sum_{i=1}^{n} s_{i}(\theta) \ln \left(\frac{n+1}{n+1-R_{+, i}^{(n)}(\theta)}\right)
$$


where $\mathcal{J}^{(n)}\left(f_{\mathcal{L}}\right):=n^{-1} \sum_{i=1}^{n}\left(\ln \left(\frac{n+1}{n+1-r}\right)\right)^{2}$. The test statistic (4.3) in this case is derived along the same lines:

$$
{\underset{\sim}{\mathcal{L}}}^{*(n)}\left(\hat{\theta}_{\#}\right):=\frac{1}{\sqrt{n \mathcal{L}^{*(n)}\left(f_{\mathcal{L}}\right)}} \sum_{i=1}^{n} s_{i}(\theta)\left(\underline{\eta}^{(n)}\left(f_{\mathcal{L}} ; \theta\right)-\ln \left(\frac{n+1}{n+1-R_{+, i}^{(n)}(\theta)}\right)\right),
$$

where $\chi^{*(n)}(\mathcal{L}):=n^{-1} \sum_{r=1}^{n}\left(\underline{\eta}^{(n)}\left(f_{\mathcal{L}} ; \theta\right)-\ln \left(\frac{n+1}{n+1-R_{+, i}^{(n)}(\theta)}\right)\right)^{2}$.

\subsection{Estimation of cross-information quantities.}

\subsubsection{Consistent estimation of $\mathcal{I}\left(f_{1}, g_{1}\right)$ and $\mathcal{M}\left(f_{1}, g_{1}\right)$.}

Implementing the rank-based tests of Section 4.3 requires consistent estimation of $\eta\left(f_{1}, g_{1}\right)$, that is, consistent estimation of the cross-information quantities $\mathcal{I}\left(f_{1}, g_{1}\right)$ and $\mathcal{M}\left(f_{1}, g_{1}\right)$. The cross-information for location $\mathcal{I}\left(f_{1}, g_{1}\right)$ is a familiar quantity in classical rank-based inference, which explicitly appears, for instance, in the asymptotic powers of traditional rank and signedrank tests for location, and in the asymptotic variance of the corresponding R-estimators. In a different context (R-estimation of shape), its counterpart also plays a central role in the construction of one-step R-estimators (Hallin, Oja, and Paindaveine 2006).

Estimating $\mathcal{I}\left(f_{1}, g_{1}\right)$ however is not straightforward. No empirical version of this expectation is available, as it involves the unknown score $\phi_{g_{1}}$ associated with the unspecified density $g_{1}$. Various methods have been proposed for estimating it. Some of them (Lehmann 1963; Sen 1966) involve comparisons of lengths of confidence intervals. Some others (Kraft and van Eeden 1972, Antille 1974, or Jurečková and Sen 1996, page 321) rely on the asymptotic linearity property of rank statistics. More elaborated approaches involve kernel estimates of $g_{1}$-hence cannot be expected to perform well under small and moderate sample sizes; they have been considered, for Wilcoxon scores, by Schweder (1975) (see also Cheng and Serfling 1981, Bickel and Ritov 1988, and Fan 1991) and, in a more general setting, in Section 4.5 of Koul (2002). All these methods however involve quite arbitrary choices (choice of a confidence level for confidence intervals; choice of an arbitrary $O\left(n^{-1 / 2}\right)$ perturbation for the method based on asymptotic linearity; choice of a kernel and of a bandwidth for the estimation of $g_{1}$ ). An unpleasant feature of all these choices is that, although they do not affect consistency and asymptotic efficiency, they have a dramatic impact on finite sample results. As for kernel methods, they are kind of antinomic to the spirit of rank-based methods: if densities are to be estimated, indeed, using them all the way by inserting estimated scores into the parametric tests of Section 3.3 seems more coherent than considering ranks.

A more sophisticated and less arbitrary way of dealing with this estimation problem was proposed (in a different context) by Hallin, Oja, and Paindaveine (2006). The basic intuition is that of solving a local linearized likelihood equation. Along with a consistent estimator of $\mathcal{I}\left(f_{1}, g_{1}\right)$, the method also provides an efficient (at $\mathrm{P}_{\theta, \sigma, 0 ; f_{1}}^{(n)}$ ) R-estimator of $\theta$, which can be used in the alignment process.

In the present setting, this estimator of $\mathcal{I}\left(f_{1}, g_{1}\right)$ is constructed as follows. Denoting by $\hat{\theta}$ and $\hat{\sigma}$ root- $n$ consistent (under $\mathrm{P}_{\theta, \sigma, 0 ; g_{1}}^{(n)}, g_{1} \in \underset{\sim}{\mathcal{F}_{f_{1}}^{*}}$ ) estimators of $\theta$ and $\sigma$, respectively, by $\hat{\theta}_{\#}$ and $\hat{\sigma}_{\#}$ their discretized versions, and by $\underset{\sim}{\Delta} f_{1} ; 1 ; \#$ a discretized version of $\underset{\sim}{(n)}(n)$ fi $f_{1}$ defined in (4.1), 
let, for any $\beta>0$,

$$
\left.{\underset{\sim}{\theta}}_{*}^{(n)}(\beta):=\hat{\theta}_{\#}+n^{-1 / 2} \beta \hat{\sigma}_{\#}^{2} \underset{\sim}{\Delta} f_{1} ; 1 ; \#\right)\left(\hat{\theta}_{\#}, \hat{\sigma}_{\#}\right) .
$$

Choosing a further arbitrary discretization constant $c>0$, put $\beta_{\ell}:=\ell / c, \ell \in \mathbb{N}$, and define

$$
\left.\beta_{1}^{-}:=\min \left\{\beta_{\ell} \mid \underset{\sim}{\Delta} f_{1} ; 1 ; \#\right)\left({\underset{\sim}{*}}_{*}^{(n)}\left(\beta_{\ell+1}\right), \hat{\sigma}_{\#}\right) \underset{\sim}{\Delta} f_{1 ; 1 ; \#}^{(n)}\left(\hat{\theta}_{\#}, \hat{\sigma}_{\#}\right)<0\right\}, \quad \beta_{1}^{+}:=\beta_{1}^{-}+\frac{1}{c},
$$

and

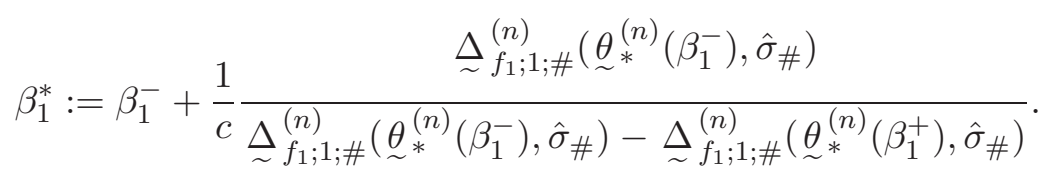

Then,

$$
\mathcal{I}^{(n)}\left(f_{1}\right):=\left(\beta_{1}^{*}\right)^{-1}=\mathcal{I}\left(f_{1}, g_{1}\right)+o_{\mathrm{P}}(1)
$$

under $\mathrm{P}_{\theta, \sigma, 0 ; g_{1}}^{(n)}, g_{1} \in{\underset{\sim}{\mathcal{F}}}_{f_{1}}^{*}$, as $n \rightarrow \infty$. Moreover, $\underset{\sim}{\theta_{f_{1}}^{(n)}}:={\underset{\sim}{\theta}}_{*}^{(n)}\left(\beta_{1}^{*}\right)$ is an efficient (at $\mathrm{P}_{\theta, \sigma, 0: f_{1}}^{(n)}$ efficiency here is in the parametric sense) R-estimator of $\theta$. A proof for this can be obtained by parallelling that of Section 4.2 in Hallin, Oja, and Paindaveine (2006). The same claim also follows from the more general result we are deriving in Proposition 5.3 of Cassart et al. (2007).

The estimation method just described for $\mathcal{I}\left(f_{1}, g_{1}\right)$ unfortunately does not apply to $\mathcal{M}\left(f_{1}, g_{1}\right)$. Contrary to $\mathcal{I}\left(f_{1}, g_{1}\right), \mathcal{M}\left(f_{1}, g_{1}\right)$ indeed is not associated with any optimal one-step R-estimation

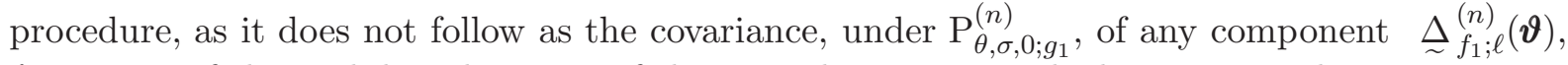
$\ell=1,2,3$, of the rank-based version of the central sequence with the corresponding component $\Delta_{g_{1} ; \ell}^{(n)}(\boldsymbol{\vartheta})$ of $\boldsymbol{\Delta}_{g_{1}}^{(n)}(\boldsymbol{\vartheta})$. Proposition 5.3 of Cassart et al. (2007) therefore develops a generalization of the Hallin, Oja, and Paindaveine (2006) method, based on a simple asymptotic linearity property.

Using the same notation as above, consider the discretized version $\underset{\sim}{\Delta}{\underset{f}{1} ; ; 3 ; \#}_{(n)}^{\left(\hat{\theta}_{\#}\right) \text { of } \underset{\sim}{\Delta} f_{1} ; 3}(n)\left(\hat{\theta}_{\#}\right)$. It follows from the proof of Proposition 6.2 that

$$
\underset{\sim}{\Delta} f_{1 ; 3}^{(n)}\left(\theta+n^{-1 / 2} t\right):=\underset{\sim}{\Delta} f_{1 ; 3}^{(n)}(\theta)+t \hat{\sigma}_{\#}^{-1} \mathcal{M}\left(f_{1}, g_{1}\right)+o_{\mathrm{P}}(1)
$$

as $n \rightarrow \infty$ under $\mathrm{P}_{\theta, \sigma, 0 ; g_{1}}^{(n)}, g_{1} \in \mathcal{F}_{\sim}^{*} f_{1}$, for all $t \in \mathbb{R}$. All other assumptions of Proposition 5.3 of Cassart et al. (2007) hold, hence also the desired consistency of the estimator we now describe.

Proceeding as in (4.4) above, let

$$
{\underset{\sim}{\theta * *}}_{*(n)}^{(n)}(\beta):=\hat{\theta}_{\#}-n^{-1 / 2} \beta \hat{\sigma}_{\#} \underset{\sim}{\Delta} f_{1} ; 3 ; \#\left(\hat{\theta}_{\#}\right),
$$

and

Defining

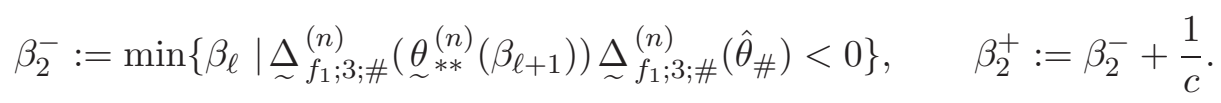

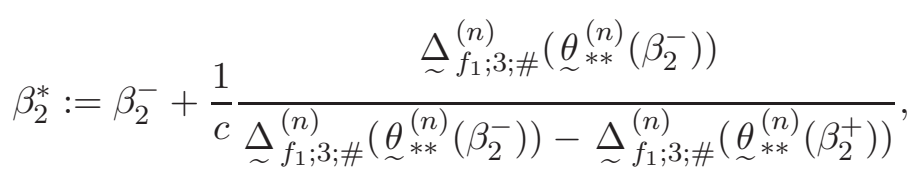

Proposition 5.3 of Cassart et al. (2007) entails

$$
\mathcal{M}^{(n)}\left(f_{1}\right):=\left(\beta_{2}^{*}\right)^{-1}=\mathcal{M}\left(f_{1}, g_{1}\right)+o_{\mathrm{P}}(1)
$$

under $\mathrm{P}_{\theta, \sigma, 0 ; g_{1}}^{(n)}, g_{1} \in \underset{\sim}{\mathcal{F}_{f_{1}}^{*}}$, as $n \rightarrow \infty$. 


\subsubsection{Practical implementation.}

Here again, all discretizations in the construction of $\mathcal{I}^{(n)}\left(f_{1}\right)$ and $\mathcal{J}^{(n)}\left(f_{1}\right)$ are required for the purpose of asymptotic results, but can be dispensed with in applications, where $n$ remains fixed; the practical versions of (4.7) and (4.9) therefore are

$$
\mathcal{I}^{(n)}\left(f_{1}\right):=\left(\beta_{1}^{*}\right)^{-1} \text { and } \mathcal{M}^{(n)}\left(f_{1}\right):=\left(\beta_{2}^{*}\right)^{-1},
$$

respectively, where

and

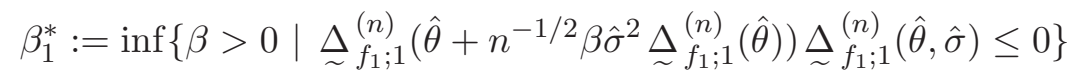

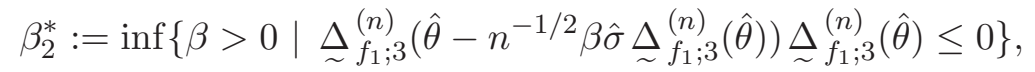

which follow from adopting "large" values of the discretizing constants (letting $c \rightarrow \infty$ ). The ratio $\mathcal{M}^{(n)}\left(f_{1}\right) / \mathcal{I}^{(n)}\left(f_{1}\right)$ then provides the estimator $\eta^{(n)}\left(f_{1} ; \theta\right)$ of $\eta\left(f_{1}, g_{1}\right)$ required in the definition (4.3) of the test statistic of Proposition 4.3.

\subsection{Asymptotic relative efficiencies.}

Propositions 4.2 and 3.6 in the specified location context and Propositions 4.3 and 3.7 in the unspecified location case allow for computing ARE values for the proposed tests based on $\underset{\sim}{T} f_{1}^{(n)}(\theta)$ and $\underset{\sim}{T} f_{1}(n) *\left(\hat{\theta}_{\#}\right)$ with respect to the pseudo-Gaussian and traditional Gaussian procedures. Those $A R E$ values are obtained as ratio of the squares of the mean values of the test statistics under local alternatives, for various densities $g_{1}$. The pseudo-Gaussian tests are not valid unless the fourth-order moments are finite, and our ARE values therefore also require the same restriction. The signed-rank tests however remain valid without such moment assumption, so that, when $g_{1}$ is such that $\mu_{4}=\infty$, their asymptotic relative efficiencies with respect to their pseudo-Gaussian counterparts can be considered as being infinite. We also obtained the ARE values for the signed-rank tests compared with the classical tests of skewness. Those ARE values will then require finite sixth-order moments.

Proposition 4.4 Let $f_{1} \in \mathcal{F}_{1}^{\square}$. Then, the asymptotic relative efficiencies, under density $g_{1} \in$ $\mathcal{F}_{\phi_{1}} \cap \underset{\sim}{\mathcal{F}} f_{1}$, of the signed-rank test based on $\underset{\sim}{T} f_{1}^{(n)}(\theta)$ with respect to the pseudo-Gaussian tests based on $\hat{T}_{\phi_{1}}^{(n)}(\theta)$, and with respect to the classical procedure based on $m_{3}^{(n)}(\theta)$, are

$$
\operatorname{ARE}_{g_{1}}\left(\underset{\sim}{f_{f_{1}}}(\theta) / \hat{T}_{\phi_{1}}^{(n)}(\theta)\right)=\left[\mathcal{L}\left(f_{1}, g_{1}\right)\right]^{2} \mu_{4} / 9 \mu_{2}^{2} \mathcal{J}\left(f_{1}\right)
$$

and

$$
\operatorname{ARE}_{g_{1}}\left(\underset{\sim}{T} f_{1}^{(n)}(\theta) / m_{3}^{(n)}(\theta)\right)=\left[\mathcal{L}\left(f_{1}, g_{1}\right)\right]^{2} \mu_{6} / 16 \mathcal{J}\left(f_{1}\right)\left[\mu_{3}^{*}\right]^{2},
$$

respectively, where the moments $\mu_{k}(\theta)$ and absolute moments $\mu_{k}^{*}(\theta)$ are computed under $g_{1}$ (equivalently, under $g$ ).

Numerical values of those AREs, under $t_{4.5}, t_{6.5}, t_{8}, t_{10}, t_{20}$, normal, logistic and doubleexponential densities, are displayed in Table 1 . Those values are good, especially when $g_{1}$ is the density of a Student with 4.5 or 6.5 degrees of freedom - which is not surprising since the ARE of the signed-rank test with respect to the pseudo-Gaussian tests based on $\hat{T}_{\phi_{1}}^{(n)}(\theta)$, under Student distributions up to four degrees of freedom are infinite, and the ARE of the signed-rank 
test with respect to the classical procedure based on $m_{3}^{(n)}(\theta)$, under Student distributions up to six degrees of freedom are infinite .

In the unspecified location case, AREs are obtained similarly for the signed-rank tests based on $\underset{\sim}{T} f_{1}(n) *\left(\hat{\theta}_{\#}\right)$, now with respect to the pseudo-Gaussian test based on $T_{\phi_{1}}^{(n) *}\left(\hat{\theta}_{\#}\right)$ and the traditional test based on $b_{1}^{(n)}$.

Proposition 4.5 Let $f_{1} \in \mathcal{F}_{1}^{\square}$. Then, the asymptotic relative efficiencies, under density $g_{1} \in$ $\mathcal{F}_{\phi_{1}} \cap \mathcal{F}_{\sim}^{\mathcal{F}_{1}}$, of the signed-rank test based on $\underset{\sim}{T_{f_{1}}^{*(n)}}\left(\hat{\theta}_{\#}\right)$ with respect to the pseudo-Gaussian tests based on $\hat{T}_{\phi_{1}}^{*(n)}\left(\hat{\theta}_{\#}\right)$, and with respect to the classical procedure based on $b_{1}^{(n)}(\hat{\theta})$, are

$$
\begin{aligned}
A R E_{g_{1}}\left({\underset{\sim}{f_{1}}}_{f_{1}}^{*(n)}\left(\hat{\theta}_{\#}\right) / \hat{T}_{\phi_{1}}^{*(n)}\left(\hat{\theta}_{\#}\right)\right) & {\left[\mathcal{L}\left(f_{1}, g_{1}\right)-\mathcal{M}\left(g_{1}, f_{1}\right) \eta\left(f_{1}, g_{1}\right)\right]^{2} /\left[\mathcal{J}\left(f_{1}\right)-2 \eta\left(f_{1}, g_{1}\right) \mathcal{M}\left(f_{1}\right)+\eta^{2}\left(f_{1}, g_{1}\right) \mathcal{I}\left(f_{1}\right)\right] } \\
& =\frac{\left[4 \mu_{1}^{* 2}-3 \mu_{2}\right]^{2} /\left[4 \mu_{1}^{* 2} \mu_{2}-4 \mu_{1}^{*} \mu_{3}^{*}+\mu_{4}\right]}{}
\end{aligned}
$$

and

$$
\begin{aligned}
& A R E_{g_{1}}\left(\underset{\sim}{T_{f_{1}}^{*(n)}}\left(\hat{\theta}_{\#}\right)\left(\hat{\theta}_{\#}\right) / b_{1}^{(n)}\right) \\
& =\frac{\left[\mathcal{L}\left(f_{1}, g_{1}\right)-\mathcal{M}\left(g_{1}, f_{1}\right) \eta\left(f_{1}, g_{1}\right)\right]^{2} /\left[\mathcal{J}\left(f_{1}\right)-2 \eta\left(f_{1}, g_{1}\right) \mathcal{M}\left(f_{1}\right)+\eta^{2}\left(f_{1}, g_{1}\right) \mathcal{I}\left(f_{1}\right)\right]}{\left[-4 \mu_{3}^{*}+6 \mu_{1}^{*} \mu_{2}\right]^{2} /\left[\mu_{6}-6 \mu_{2} \mu_{4}+9 \mu_{2}^{3}\right]},
\end{aligned}
$$

respectively, where the moments $\mu_{k}(\theta)$ and absolute moments $\mu_{k}^{*}(\theta)$ are computed under $g_{1}$ (equivalently, under $g$ ).

Numerical values of those AREs are displayed in Table 2. Again, those values are good, especially for Student densities $g_{1}$ with low degrees of freedom. The ARE values of the tests based on $\underset{\sim}{T} \phi_{1}^{*(n)}$ are uniformly larger than or equal to one.

\subsection{Simulation results.}

In order to investigate the finite-sample performance of the proposed procedures, we generated $N=5,000$ independent samples of size $n=500$ from the skewed normal (hereafter denoted as $\mathcal{S N}(\xi)$ ) and skewed Student (with $\nu=3$ and $\nu=8$ degrees of freedom, denoted as $\mathcal{S} t(\nu, \xi)$ ) densities, as defined in (1.2), for $\xi=0,0.05$, and 0.1 (recall that $\xi=0$ means symmetry). Each sample was subjected to the following fixed- $\theta$ tests for symmetry, at asymptotic level $\alpha=5 \%$ : the classical test of skewness, based on $m_{3}^{(n)}(\theta)$, the (optimal) pseudo-Gaussian test based on $T_{\phi_{1}}^{(n) *}(\theta, \hat{\sigma})$, the van der Waerden test (Section 4.4), two $t_{\nu^{-}}$score tests $(\nu=3$ and 8 ), and the Wilcoxon test (Section 4.4). Without loss of generality, $\theta$ was set to zero.

Similarly, we generated $N=5,000$ independent samples of size $n=100$ from the same skewed normal and skewed Student densities. These samples were subjected to unspecified- $\theta$ tests for symmetry, at asymptotic level $\alpha=5 \%$ : the classical test of skewness, based on $b_{1}^{(n)}$, the (optimal) pseudo-Gaussian test based on $T_{\phi_{1}}^{(n) *}(\hat{\theta}, \hat{\sigma})$, the van der Waerden test (Section 4.4),

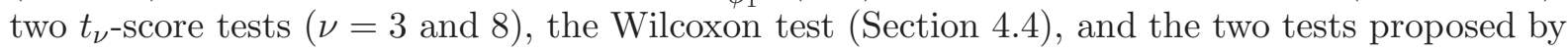




\begin{tabular}{|c|c|c|c|c|c|c|c|c|c|c|c|}
\hline & \multicolumn{11}{|c|}{$g_{1}$} \\
\hline score $f_{1}$ & $f_{t_{4.5}}$ & $f_{t_{6.5}}$ & $f_{t_{8}}$ & $f_{t_{10}}$ & $f_{t_{20}}$ & $\phi_{1}$ & $f_{\mathcal{E}_{2}}$ & $f_{\mathcal{E}_{3}}$ & $f_{\mathcal{E}_{5}}$ & $f_{\text {Log }}$ & $\overline{f_{\mathcal{L}}}$ \\
\hline \multirow{2}{*}{$f_{t_{4.5}}$} & 3.6667 & 1.4148 & 1.2156 & 1.1103 & 0.9910 & 0.9341 & 0.9646 & 1.0608 & 1.2271 & 1.1185 & 1.3086 \\
\hline & $\infty$ & 9.4990 & 3.0391 & 1.9986 & 1.2906 & 1.0316 & 0.8262 & 0.9402 & 0.9194 & 1.8164 & 2.4536 \\
\hline \multirow[t]{2}{*}{$f_{t_{6.5}}$} & 3.6505 & 1.4211 & 1.2261 & 1.1241 & 1.0116 & 0.9622 & 1.0261 & 1.1532 & 1.3749 & 1.1299 & 1.3142 \\
\hline & $\infty$ & 9.5410 & 3.0652 & 2.0234 & 1.3174 & 1.0628 & 0.8788 & 0.9134 & 1.0302 & 1.8350 & 2.4642 \\
\hline \multirow[t]{2}{*}{$f_{t_{8}}$} & 3.6319 & 1.4197 & 1.2273 & 1.1273 & 1.0184 & 0.9730 & 1.0527 & 1.1949 & 1.4445 & 1.1322 & 1.3138 \\
\hline & $\infty$ & 9.5317 & 3.0682 & 2.0291 & 1.3263 & 1.0747 & 0.9016 & 0.9464 & 1.0824 & 1.8386 & 2.4634 \\
\hline \multirow[t]{2}{*}{$f_{t_{10}}$} & 3.6086 & 1.4159 & 1.2263 & 1.1282 & 1.0130 & 0.9815 & 1.0758 & 1.2320 & 1.5082 & 1.1325 & 1.3116 \\
\hline & $\infty$ & 9.5066 & 3.0656 & 2.0308 & 1.3323 & 1.0840 & 0.9214 & 0.9759 & 1.1301 & 1.8391 & 2.4592 \\
\hline \multirow[t]{2}{*}{$f_{t_{20}}$} & 3.5375 & 1.3995 & 1.2168 & 1.1236 & 1.0172 & 0.9946 & 1.1205 & 1.3077 & 1.6440 & 1.1270 & 1.3006 \\
\hline & $\infty$ & 9.3964 & 3.0421 & 2.0226 & 1.3377 & 1.0986 & 0.9596 & 1.0358 & 1.2318 & 1.8302 & 2.4386 \\
\hline \multirow[t]{2}{*}{$\phi_{1}$} & 3.4249 & 1.3674 & 1.1942 & 1.1073 & 1.0217 & 1 & 1.1600 & 1.3813 & 1.7868 & 1.104 & 0.9634 \\
\hline & $\infty$ & 9.1808 & 2.9855 & 1.9932 & 1.3305 & 1.1045 & 0.9935 & 1.0941 & 1.3388 & 1.8033 & 1.8065 \\
\hline \multirow[t]{2}{*}{$f_{\mathcal{E}_{2}}$} & 2.9092 & 1.1993 & 1.0626 & 0.9982 & 0.9466 & 0.9541 & 1.2158 & 1.5298 & 2.1148 & 0.9936 & 1.1158 \\
\hline & $\infty$ & 8.0521 & 2.6566 & 1.7968 & 1.2328 & 1.0537 & 1.0413 & 1.2117 & 1.5846 & 1.6135 & 2.0922 \\
\hline \multirow[t]{2}{*}{$f_{\mathcal{E}_{3}}$} & 2.5003 & 1.0535 & 0.9427 & 0.8936 & 0.8635 & 0.8880 & 1.1957 & 1.5556 & 2.2418 & 0.8868 & 0.9878 \\
\hline & $\infty$ & 7.0731 & 2.3568 & 1.6084 & 1.1245 & 0.9808 & 1.0240 & 1.2321 & 1.6798 & 1.4402 & 1.8522 \\
\hline \multirow[t]{2}{*}{$f_{\mathcal{E}_{5}}$} & 1.9538 & 0.8484 & 0.7698 & 0.7389 & 0.7333 & 0.7759 & 1.1165 & 1.5143 & 2.3029 & .7328 & 0.8134 \\
\hline & $\infty$ & 5.6965 & 1.9246 & 1.3300 & 0.9549 & 0.8570 & 0.9562 & 1.1995 & 1.7255 & 1.1901 & 1.5252 \\
\hline \multirow[t]{2}{*}{$f_{\text {Log }}$} & 3.6166 & 1.4160 & 1.2253 & 1.1267 & 1.0208 & 0.9792 & 1.0653 & 1.2165 & 1.4882 & 1.1340 & 1.3196 \\
\hline & $\infty$ & 9.5068 & 3.0633 & 2.0281 & 1.3294 & 1.0815 & 0.9124 & 0.9636 & 1.1151 & 1.8415 & 2.4743 \\
\hline \multirow[t]{2}{*}{$f_{\mathcal{L}}$} & 3.5986 & 1.4007 & 1.2093 & 1.1098 & 1.0019 & 0.9581 & 1.0175 & 1.1525 & 1.4049 & 1.1223 & 1.3333 \\
\hline & $\infty$ & 9.4043 & 3.0232 & 1.9976 & 1.3048 & 1.0582 & 0.8714 & 0.9128 & 1.0527 & 1.8226 & 2.5 \\
\hline
\end{tabular}

Table 1: AREs, under various densities $g_{1}$, of the signed-rank tests based on $\underset{\sim}{T} f_{1}^{(n)}(\theta)$ with respect to the pseudo-Gaussian test based on $\hat{T}_{\phi_{1}}^{(n)}(\theta)$ (first line), and with respect to the classical procedure based on $m_{3}^{(n)}(\theta)$ (second line) for testing symmetry about a specified center $\theta$. 


\begin{tabular}{|c|c|c|c|c|c|c|c|c|c|c|c|}
\hline & \multicolumn{11}{|c|}{$g_{1}$} \\
\hline score $f_{1}$ & $f_{t_{4.5}}$ & $f_{t_{6.5}}$ & $f_{t_{8}}$ & $f_{t_{10}}$ & $f_{t_{20}}$ & $\phi_{1}$ & $f_{\mathcal{E}_{2}}$ & $f_{\mathcal{E}_{3}}$ & $f_{\mathcal{E}_{5}}$ & $f_{\log }$ & $f_{\mathcal{L}}$ \\
\hline \multirow[t]{2}{*}{$f_{t_{4.5}}$} & 6.5061 & 1.7670 & 1.3894 & 1.1993 & 1.0010 & 0.9257 & 1.0994 & 1.5174 & 2.4838 & 1.1815 & 1.6830 \\
\hline & $\infty$ & 10.5569 & 3.2004 & 2.0268 & 1.2483 & 0.9891 & 0.8227 & 1.0354 & 1.5907 & 1.7409 & 2.9453 \\
\hline \multirow[t]{2}{*}{$f_{t_{6.5}}$} & 6.4689 & 1.7771 & 1.4047 & 1.2182 & 1.0265 & 0.9584 & 1.2131 & 1.7323 & 2.9581 & 1.1961 & 1.6550 \\
\hline & $\infty$ & 10.6176 & 3.2357 & 2.0587 & 1.2801 & 1.0241 & 0.9078 & 1.1820 & 1.8944 & 1.7624 & 2.8963 \\
\hline \multirow[t]{2}{*}{$f_{t_{8}}$} & 6.4272 & 1.7750 & 1.4064 & 1.2223 & 1.0347 & 0.9706 & 1.2637 & 1.8313 & 3.1830 & 1.1987 & 1.6385 \\
\hline & $\infty$ & 10.6047 & 3.2396 & 2.0657 & 1.2903 & 1.0371 & 0.9457 & 1.2495 & 2.0384 & 1.7663 & 2.8674 \\
\hline \multirow[t]{2}{*}{$f_{t_{10}}$} & 6.3759 & 1.7692 & 1.4050 & 1.2236 & 1.0402 & 0.9799 & 1.3087 & 1.9212 & 3.3908 & 1.1988 & 1.6214 \\
\hline & $\infty$ & 10.5704 & 3.2363 & 2.0677 & 1.2971 & 1.0471 & 0.9793 & 1.3109 & 2.1715 & 1.7664 & 2.8375 \\
\hline \multirow[t]{2}{*}{$f_{t_{20}}$} & 6.2234 & 1.7446 & 1.3922 & 1.2178 & 1.0453 & 0.9943 & 1.4007 & 2.1124 & 3.8450 & 1.1913 & 1.5778 \\
\hline & $\infty$ & 10.4234 & 3.2068 & 2.0579 & 1.3032 & 1.0625 & 1.0482 & 1.4413 & 2.4623 & 1.7553 & 2.7611 \\
\hline \multirow[t]{2}{*}{$\phi_{1}$} & 5.9895 & 1.6979 & 1.3622 & 1.1975 & 1.0388 & 1 & 1.4932 & 2.3174 & 4.3526 & 1.1706 & 1.0750 \\
\hline & $\infty$ & 10.1440 & 3.1377 & 2.0237 & 1.2954 & 1.0686 & 1.1174 & 1.5812 & 2.7874 & 1.7247 & 1.8812 \\
\hline \multirow[t]{2}{*}{$f_{\mathcal{E}_{2}}$} & 4.0839 & 1.1987 & 0.9789 & 0.8753 & 0.7891 & 0.7941 & 1.9908 & 4.1088 & 10.1249 & 0.8465 & 0.9022 \\
\hline & $\infty$ & 7.1619 & 2.2548 & 1.4792 & 0.9840 & 0.8485 & 1.4897 & 2.8035 & 6.4839 & 1.2473 & 1.5789 \\
\hline \multirow[t]{2}{*}{$f_{\mathcal{E}_{3}}$} & 2.9971 & 0.8871 & 0.7278 & 0.6538 & 0.5963 & 0.6089 & 1.8544 & 4.4376 & 13.0663 & 0.6331 & 0.6354 \\
\hline & $\infty$ & 5.3001 & 1.6765 & 1.1050 & 0.7436 & 0.6506 & 1.3877 & 3.0278 & 8.3677 & 0.9329 & 1.1120 \\
\hline \multirow[t]{2}{*}{$f_{\mathcal{E}_{5}}$} & 1.9423 & 0.5779 & 0.4756 & 0.4287 & 0.3943 & 0.4072 & 1.4549 & 4.0156 & 14.5550 & 0.4164 & 0.3981 \\
\hline & $\infty$ & 3.4528 & 1.0956 & 0.7244 & 0.4916 & 0.4351 & 1.0887 & 2.7399 & 9.3210 & 0.6136 & 0.6967 \\
\hline \multirow[t]{2}{*}{$f_{\text {Log }}$} & 6.3951 & 1.7696 & 1.4036 & 1.2211 & 1.0361 & 0.9745 & 1.2444 & 1.7778 & 3.0370 & 1.2012 & 1.6523 \\
\hline & $\infty$ & 10.5724 & 3.2331 & 2.0636 & 1.2920 & 1.0413 & 0.9312 & 1.2130 & 1.9449 & 1.7699 & 2.8915 \\
\hline \multirow[t]{2}{*}{$f_{\mathcal{L}}$} & 4.9601 & 1.2903 & 0.9964 & 0.8468 & 0.6862 & 0.6177 & 0.4604 & 0.5579 & 0.8512 & 0.8681 & 2 \\
\hline & $\infty$ & 7.7087 & 2.2950 & 1.4311 & 0.8557 & 0.6601 & 0.3445 & 0.3807 & 0.5451 & 1.2791 & 3.5 \\
\hline
\end{tabular}

Table 2: AREs, under various densities $g_{1}$, of the signed-rank tests based on $\underset{\sim}{T} f_{1}^{*(n)}\left(\hat{\theta}_{\#}\right)$ with respect to the pseudo-Gaussian test based on $\hat{T}_{\phi_{1}}^{*(n)}\left(\hat{\theta}_{\#}\right)$ (first line), and with respect to the classical procedure based on $b_{1}^{(n)}$ (second line) for testing symmetry about unspecified $\theta$. 
Randles et al. (1980), which are based on the signs of $X_{i}+X_{j}-2 X_{k}, 1 \leq i<j<k \leq n$. All tests were performed under two-sided form; rejection frequencies are reported in Tables 3 and 4 .

Inspection of those tables reveals that classical tests, whether based on $m_{3}^{(n)}(\theta)$ or on $b_{1}^{(n)}$, are prohibitively conservative under $t_{3}$, and biased or very weak under the related $\mathcal{S} t(3, \xi)$ densities, which can be explained by infinite third-order moments. The same weaknesses are still observed under $t_{8}$ and $\mathcal{S} t(8, \xi)$ densities, despite the existence of moments of order six. Those classical tests are hardly recommendable, thus, when heavier-than-normal tails are possible. The pseudo-Gaussian tests based on $T_{\phi_{1}}^{(n)}(\theta, \hat{\sigma})$ and $T_{\phi_{1}}^{(n) *}(\hat{\theta}, \hat{\sigma})$ perform quite reasonably, and uniformly better than those based on $m_{3}^{(n)}(\theta)$ or $b_{1}^{(n)}$. The signed-rank tests based on $\underset{\sim}{T} f_{1}^{(n)}(\theta)$ and $\underset{\sim}{T_{f_{1}}}{ }^{(n)}(\hat{\theta})$ all satisfy the nominal level constraint, and their rejection frequencies are in line with the asymptotic relative efficiencies of Table 1 . It is to be noted that, under $\mathcal{S} t(8, \xi), \underset{\sim}{t_{t_{8}}^{(n)}}(\theta)$ and $\underset{\sim}{T} f_{\text {Log }}^{(n)}(\theta)$ achieve similar performances, reflecting the closeness of their respective AREs.

The unspecified- $\theta$ rank-based tests however all are quite conservative (their rejection frequencies under the null are significantly less than 5\%) when asymptotic critical values are used. This undesirable feature of course has a negative impact on the corresponding powers. In order to raise the power curves, simulated critical values were computed; the resulting rejection frequencies are also shown in Table 4 . The 5\% probability level constraint apparently is met, although the test statistics $\underset{\sim}{T} f_{1}^{*(n)}(\hat{\theta})$ are only asymptotically distribution-free under the null, so that those simulated critical values are only an approximation.

In this context of relatively small sample sizes, observed rejection frequencies are not completely in line with the asymptotic relative efficiency results of Table 2. The tests of Randles et al. yield high rejection frequencies, in particular under $\mathcal{S} t(\nu, \xi)$ densities, but in several cases fail to satisfy the $5 \%$ level constraint (recall that they are not distribution-free).

\begin{tabular}{|c|c|c|c|c|c|c|c|c|c|}
\hline & \multicolumn{3}{|c|}{$\overline{\mathcal{S N}(\xi)}$} & \multicolumn{3}{|c|}{$\overline{\mathcal{S} t(3, \xi)}$} & \multicolumn{3}{|c|}{$\mathcal{S} t(8, \xi)$} \\
\hline Test & & $\xi$ & & & $\xi$ & & & $\xi$ & \\
\hline & 0 & 0.05 & 0.1 & 0 & 0.05 & 0.1 & 0 & 0.05 & 0.1 \\
\hline$m_{3}^{(n)}(\theta)$ & 0.0476 & 0.4526 & 0.9594 & 0.0202 & 0.0498 & 0.1726 & 0.0388 & 0.2472 & 0.7096 \\
\hline$\hat{T}_{\phi_{1}}^{(n)}(\theta)$ & 0.0452 & 0.4882 & 0.9734 & 0.0358 & 0.1398 & 0.4346 & 0.0456 & 0.3626 & 0.8954 \\
\hline$T_{t_{3}}^{(n)}(\theta)$ & 0.0454 & 0.4512 & 0.9518 & 0.0464 & 0.3456 & 0.8824 & 0.0466 & 0.4010 & 0.9288 \\
\hline${\underset{\sim}{T} t_{8}^{(n)}(\theta)}^{(n)}$ & 0.0460 & 0.4784 & 0.9668 & 0.0482 & 0.3352 & 0.8702 & 0.0442 & 0.4086 & 0.9376 \\
\hline$\widetilde{\sim}_{\phi_{1}}^{(n)}(\theta)$ & 0.0466 & 0.4858 & 0.9708 & 0.0494 & 0.3224 & 0.8486 & 0.0440 & 0.4062 & 0.9356 \\
\hline${\stackrel{\sim}{\sim} f_{\text {Log }}^{(n)}(\theta)}_{(n)}^{(n)}$ & 0.0456 & 0.4810 & 0.9668 & 0.0494 & 0.3342 & 0.8696 & 0.0434 & 0.4076 & 0.9382 \\
\hline
\end{tabular}

Table 3: Rejection frequencies (out of $N=5,000$ replications), under various symmetric and related skewed densities $(1.2), \mathcal{S N}(\xi)$ and $\mathcal{S} t(\nu, \xi)(\nu=3,8$ and $\xi=0,0.05,0.1)$, of (1) the classical tests of skewness, based on $m_{3}^{(n)}(\theta),(2)$ the van der Waerden test, $t_{\nu}$-score tests $(\nu=3$ and 8$)$, and the Wilcoxon test, all for testing symmetry with respect to specified $(\theta=0)$ location.

Besides their performance in Fechner family, we also investigated the finite-sample behaviour of the proposed procedures within the families of skewed distributions recently introduced by Azzalini and Capitanio (2003), which are not of the Fechner type. We generated $N=5,000$ independent samples of size $n=100$ from the skewed normal (hereafter denoted as $\mathcal{A S N}(\xi)$ ) 


\begin{tabular}{|c|ccc|ccc|ccc|}
\hline & \multicolumn{3}{|c|}{$\mathcal{S N}(\xi)$} & \multicolumn{3}{c|}{$\mathcal{S} t(3, \xi)$} & \multicolumn{3}{c|}{$\mathcal{S} t(8, \xi)$} \\
\hline Test & \multicolumn{3}{|c|}{$\xi$} & & \multicolumn{3}{c|}{$\xi$} & & \multicolumn{3}{c|}{$\xi$} \\
\hline & 0 & 0.3 & 0.6 & 0 & 0.3 & 0.6 & 0 & 0.3 & 0.6 \\
\hline$b_{1}^{(n)}$ & 0.0410 & 0.3796 & 0.8374 & 0.0244 & 0.1806 & 0.4188 & 0.0368 & 0.3252 & 0.7070 \\
\hline$\hat{T}_{\phi_{1}}^{*(n)}(\hat{\theta})$ & 0.0448 & 0.4228 & 0.8876 & 0.0300 & 0.2858 & 0.6326 & 0.0458 & 0.4294 & 0.8580 \\
\hline${\underset{\sim}{\sim}}_{t_{3}}^{*(n)}(\hat{\theta})$ & 0.0460 & 0.3658 & 0.8192 & 0.0390 & 0.5232 & 0.9664 & 0.0412 & 0.4414 & 0.9028 \\
& 0.0484 & 0.3786 & 0.8266 & 0.0422 & 0.5376 & 0.9692 & 0.0456 & 0.4570 & 0.9076 \\
\hline${\underset{\sim}{\sim}}_{t_{8}}^{*(n)}(\hat{\theta})$ & 0.0412 & 0.3872 & 0.8664 & 0.0366 & 0.5294 & 0.9814 & 0.0424 & 0.4620 & 0.9352 \\
& 0.0498 & 0.4204 & 0.8816 & 0.0456 & 0.5640 & 0.9850 & 0.0522 & 0.4956 & 0.9450 \\
\hline${\underset{\sim}{\sim}}_{\phi_{1}}^{*(n)}(\hat{\theta})$ & 0.0386 & 0.3850 & 0.8812 & 0.0368 & 0.5156 & 0.9794 & 0.0418 & 0.4580 & 0.9452 \\
& 0.0528 & 0.4294 & 0.9044 & 0.0486 & 0.5690 & 0.9854 & 0.0540 & 0.5116 & 0.9558 \\
\hline${\underset{\sim}{\sim} f_{L o g}^{*(n)}(\hat{\theta})}^{0.0420}$ & 0.3868 & 0.8548 & 0.0378 & 0.5314 & 0.9778 & 0.0418 & 0.4566 & 0.9318 \\
& 0.0488 & 0.4168 & 0.8718 & 0.0456 & 0.5652 & 0.9818 & 0.0504 & 0.4910 & 0.9410 \\
\hline$T_{R 1}$ & 0.0504 & 0.4444 & 0.9006 & 0.0562 & 0.6048 & 0.9916 & 0.0588 & 0.5332 & 0.9640 \\
$T_{R 2}$ & 0.0576 & 0.4636 & 0.9050 & 0.0602 & 0.6196 & 0.9926 & 0.0644 & 0.5534 & 0.9662 \\
\hline
\end{tabular}

Table 4: Rejection frequencies (out of $N=5,000$ replications), under various symmetric and related skewed densities $(1.2), \mathcal{S N}(\xi)$ and $\mathcal{S} t(\nu, \xi)(\nu=3,8$ and $\xi=0,0.3,0.6)$, of (1) the classical tests of skewness, based on $b_{1}^{(n)},(2)$ the van der Waerden test, $t_{\nu}$-score tests $(\nu=3$ and 8), and the Wilcoxon test, based on asymptotic critical values (first line) and simulated critical values (second line), and (3) the tests of Randles et al. (1980).

and skewed Student (with $\nu=3$ and $\nu=8$ degrees of freedom, denoted as $\mathcal{A S t}(\nu, \xi)$ ) densities, as defined in Azzalini and Capitanio (2003), for $\xi=0,3$, and 6 ( $\xi=0$ means symmetry). Each sample was subjected to the following fixed- $\theta$ tests for symmetry, at asymptotic level $\alpha=5 \%$ : the classical test of skewness, based on $m_{3}^{(n)}(\theta)$, the pseudo-Gaussian test based on $T_{\phi_{1}}^{(n) *}(\theta, \hat{\sigma})$, the van der Waerden test (Section 4.4), two $t_{\nu}$-score tests $(\nu=3$ and 8$)$, and the Wilcoxon test (Section 4.4). Without loss of generality, $\theta$ was set to zero. Prior to performing fixed-location procedures, we centered the sample at the population median (this median was computed numerically by simulating a sample of 10,000 independent observations).

Similarly, we generated $N=5,000$ independent samples of size $n=100$ from the same skewed normal and skewed Student densities. These samples were subjected to unspecified- $\theta$ tests for symmetry, at asymptotic level $\alpha=5 \%$ : the classical test of skewness, based on $b_{1}^{(n)}$, the (optimal) pseudo-Gaussian test based on $T_{\phi_{1}}^{(n) *}(\hat{\theta}, \hat{\sigma})$, the van der Waerden test (Section 4.4),

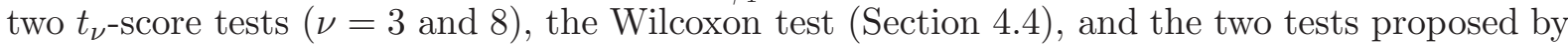
Randles et al. (1980), which are based on the signs of $X_{i}+X_{j}-2 X_{k}, 1 \leq i<j<k \leq n$. All tests were performed under two-sided form; rejection frequencies are reported in Tables 5 and 6 .

Inspection of those tables reveals that classical tests, whether based on $m_{3}^{(n)}(\theta)$ or on $b_{1}^{(n)}$, are prohibitively conservative under $t_{3}$, which can be explained by infinite third-order moments, and exhibit very weak performance under the related $\mathcal{S} t(3, \xi)$ densities. The pseudo-Gaussian tests based on $T_{\phi_{1}}^{(n)}(\theta, \hat{\sigma})$ and $T_{\phi_{1}}^{(n) *}(\hat{\theta}, \hat{\sigma})$ perform quite reasonably, and uniformly better than those based on $m_{3}^{(n)}(\theta)$ or $b_{1}^{(n)}$. The signed-rank tests based on $\underset{\sim}{T} f_{1}^{(n)}(\theta)$ and $\underset{\widetilde{f}}{T} f_{1}^{*(n)}(\hat{\theta})$ all satisfy the nominal level constraint and perform reasonably well in this situation for which they are 
not optimal. Here again, the rank-based tests however all are quite conservative (their rejection frequencies under the null are significantly less than 5\%) when asymptotic critical values are used. Using simulated critical values raises the power curves without affecting type I risk, as can be noticed in Table 5 and Table 6 . The tests of Randles et al. yield high rejection frequencies, in particular under $\mathcal{S} t(\nu, \xi)$ densities, but in several cases fail to satisfy the $5 \%$ level constraint.

\begin{tabular}{|c|c|c|c|c|c|c|c|c|c|}
\hline & \multicolumn{3}{|c|}{$\mathcal{S \mathcal { N }}(\xi)$} & \multicolumn{3}{|c|}{$\mathcal{S} t(3, \xi)$} & \multicolumn{3}{|c|}{$\mathcal{S} t(8, \xi)$} \\
\hline \multirow{2}{*}{ Test } & \multicolumn{3}{|c|}{$\xi$} & \multicolumn{3}{|c|}{$\xi$} & \multicolumn{3}{|c|}{$\xi$} \\
\hline & 0 & 0.05 & 0.1 & 0 & 0.05 & 0.1 & 0 & 0.05 & 0.1 \\
\hline$m_{3}^{(n)}(\theta)$ & 0.0396 & 0.5592 & 0.8274 & 0.0152 & 0.0552 & 0.1036 & 0.0278 & 0.2704 & 0.4994 \\
\hline$\hat{T}_{\phi_{1}}^{(n)}(\theta)$ & 0.0420 & 0.4886 & 0.7710 & 0.0258 & 0.1034 & 0.1736 & 0.0382 & 0.3034 & 0.5560 \\
\hline \multirow[t]{2}{*}{${\underset{\sim}{T}}_{t_{3}}^{(n)}(\theta)$} & 0.0434 & 0.2570 & 0.4514 & 0.0470 & 0.1284 & 0.1860 & 0.0442 & 0.1866 & 0.3316 \\
\hline & 0.0452 & 0.2612 & 0.4552 & 0.0486 & 0.1314 & 0.1878 & 0.0462 & 0.1896 & 0.3350 \\
\hline \multirow[t]{2}{*}{$T_{\sim}^{T_{t_{8}}^{(n)}(\theta)}$} & 0.0426 & 0.3590 & 0.6066 & 0.0462 & 0.1446 & 0.2282 & 0.0424 & 0.2450 & 0.4426 \\
\hline & 0.0434 & 0.3644 & 0.6100 & 0.0478 & 0.1474 & 0.2334 & 0.0440 & 0.2482 & 0.4476 \\
\hline \multirow{2}{*}{$\underset{\sim}{T} \phi_{1}^{(n)}(\theta)$} & 0.0434 & 0.4286 & 0.6952 & 0.0448 & 0.1504 & 0.2454 & 0.0422 & 0.2772 & 0.5056 \\
\hline & 0.0440 & 0.4354 & 0.7006 & 0.0468 & 0.1532 & 0.2500 & 0.0440 & 0.2832 & 0.5120 \\
\hline \multirow[t]{2}{*}{${\underset{\sim}{\sim} f_{\text {Log }}^{(n)}(\theta)}^{(n)}$} & 0.0424 & 0.3444 & 0.5862 & 0.0464 & 0.1408 & 0.2222 & 0.0430 & 0.2370 & 0.4274 \\
\hline & 0.0432 & 0.3484 & 0.5898 & 0.0480 & 0.1438 & 0.2252 & 0.0436 & 0.2394 & 0.4320 \\
\hline
\end{tabular}

Table 5: Rejection frequencies (out of $N=5,000$ replications), under various symmetric and related skewed densities, $\mathcal{A S N}(\xi)$ and $\mathcal{A S t}(\nu, \xi)(\nu=3,8$ and $\xi=0,0.05,0.1)$, of (1) the

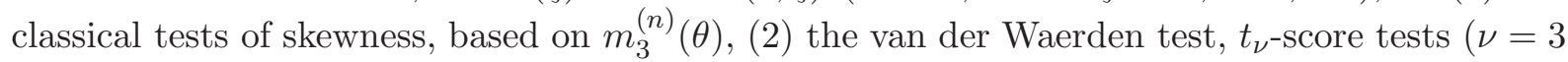
and 8 ), and the Wilcoxon test, based on asymptotic critical values (first line) and simulated critical values (second line), all for testing symmetry with respect to specified $(\theta=0)$ location.

\section{Future research}

The problems and the methods described here all are inherently univariate. A natural and useful multivariate extension of the hypothesis of symmetry is that of elliptical symmetry. In an ongoing research, we propose to extend the results of this paper to the problem of testing for elliptical symmetry (with respect to specified or unspecified location $\boldsymbol{\theta}$, and with unspecified scatter matrix $\boldsymbol{\Sigma}$ ). We therefore propose a multivariate extension of the Fechner families, with densities of the form

$$
\frac{1}{|\boldsymbol{\Sigma}|^{1 / 2}} f_{1}\left(\left((\boldsymbol{x}-\boldsymbol{\theta})^{\prime} \boldsymbol{\Sigma}^{-1 / 2} \boldsymbol{B}_{\boldsymbol{\Sigma}^{-1 / 2}(\boldsymbol{x}-\boldsymbol{\theta})}^{-2} \boldsymbol{\Sigma}^{-1 / 2}(\boldsymbol{x}-\boldsymbol{\theta})\right)^{1 / 2}\right), \boldsymbol{x} \in \mathbb{R}^{k}
$$

where $\boldsymbol{\theta} \in \mathbb{R}^{k}$ is a location parameter, $\boldsymbol{\Sigma} \in \mathcal{S}_{k}:=\left\{\mathbf{M} \in \mathbb{R}^{k \times k} \mid \mathbf{M}\right.$ is symmetric and positive definite $\}$ is a scatter matrix, $\boldsymbol{\Sigma}^{1 / 2}$ is the symmetric square root of $\boldsymbol{\Sigma}$, the matrix $\mathbf{B}_{\mathbf{Z}}$ is diagonal with $\left(\mathbf{B}_{\mathbf{Z}}\right)_{j j}:=\left(1-\operatorname{sgn}\left(Z_{j}\right) \xi_{j}\right)$ and $\boldsymbol{\xi}=\left(\xi_{1}, \ldots, \xi_{k}\right)^{\prime} \in(-1,1)^{k}$ is the asymmetry parameter. The infinite-dimensional parameter $f_{1}$ is an a.e. strictly positive function from $\mathbb{R}_{0}^{+}$to $\mathbb{R}^{+}$, called a radial density. 


\begin{tabular}{|c|ccc|ccc|ccc|}
\hline & \multicolumn{3}{|c|}{$\mathcal{S \mathcal { N }}(\lambda)$} & \multicolumn{3}{c|}{$\mathcal{S} t(3, \lambda)$} & \multicolumn{3}{c|}{$\mathcal{S} t(8, \lambda)$} \\
\hline Test & \multicolumn{3}{|c|}{$\lambda$} & \multicolumn{3}{c|}{$\lambda$} & 0 & \multicolumn{3}{c|}{$\lambda$} \\
\hline & 0 & 3 & 6 & 0 & 3 & 6 & 0 & 3 & 6 \\
\hline$b_{1}^{(n)}$ & 0.0410 & 0.6222 & 0.8740 & 0.0244 & 0.0670 & 0.1210 & 0.0368 & 0.3076 & 0.5510 \\
$\hat{T}_{\phi_{1}^{*}(n)}^{(n)}$ & 0.0448 & 0.6992 & 0.9382 & 0.0300 & 0.1122 & 0.1982 & 0.0458 & 0.4064 & 0.7038 \\
\hline${\underset{\sim}{\sim}}_{t_{3}}^{*(n)}(\hat{\theta})$ & 0.0460 & 0.6042 & 0.8958 & 0.0390 & 0.2154 & 0.3974 & 0.0412 & 0.4322 & 0.7456 \\
& 0.0484 & 0.6162 & 0.9032 & 0.0422 & 0.2254 & 0.4134 & 0.0456 & 0.4448 & 0.7544 \\
\hline$\underset{\sim}{T} t_{8}^{*(n)}(\hat{\theta})$ & 0.0412 & 0.6516 & 0.9294 & 0.0366 & 0.2138 & 0.3826 & 0.0424 & 0.4476 & 0.7752 \\
& 0.0498 & 0.6868 & 0.9394 & 0.0456 & 02368 & 0.4176 & 0.0522 & 0.4812 & 0.8028 \\
\hline$\underset{\sim}{T} \phi_{1}^{*(n)}(\hat{\theta})$ & 0.0386 & 0.6700 & 0.9374 & 0.0368 & 0.20006 & 0.3598 & 0.0418 & 0.4436 & 0.7714 \\
& 0.0528 & 0.7104 & 0.9492 & 0.0486 & 0.2360 & 0.4030 & 0.0540 & 0.4918 & 0.8070 \\
\hline${\underset{T}{\sim}}_{f_{\text {Log }}^{*(n)}(\hat{\theta})}$ & 0.0420 & 0.6490 & 0.9230 & 0.0378 & 0.2146 & 0.3898 & 0.0418 & 0.4462 & 0.7716 \\
& 0.0488 & 0.6820 & 0.9338 & 0.0456 & 0.2390 & 0.4188 & 0.0504 & 0.4462 & 0.7716 \\
\hline$T_{R 1}$ & 0.0504 & 0.4444 & 0.9006 & 0.0562 & 0.6048 & 0.9916 & 0.0588 & 0.5332 & 0.9640 \\
$T_{R 2}$ & 0.0576 & 0.4636 & 0.9050 & 0.0602 & 0.6196 & 0.9926 & 0.0644 & 0.5534 & 0.9662 \\
\hline
\end{tabular}

Table 6: Rejection frequencies (out of $N=5,000$ replications), under various symmetric and related skewed densities, $\mathcal{A S N}(\xi)$ and $\mathcal{A S t}(\nu, \xi)(\nu=3,8$ and $\xi=0,0.3,0.6)$, of (1) the classical tests of skewness, based on $b_{1}^{(n)},(2)$ the van der Waerden test, $t_{\nu}$-score tests $(\nu=3$ and 8$)$, and the Wilcoxon test, based on asymptotic critical values (first line) and simulated critical values (second line), and (3) the tests of Randles et al. (1980).

\section{Appendix.}

\subsection{Proof of Proposition 3.1.}

The proof of Proposition 3.1 relies on Swensen (1985)'s Lemma 1. The sufficient conditions for LAN given there follow from standard arguments. The main point consists in showing that $(\theta, \sigma, \xi) \mapsto f_{\theta, \sigma, \xi ; f_{1}}^{1 / 2}(x)$, where $f_{\theta, \sigma, \xi ; f_{1}}$ is the density in (3.1), is differentiable in quadratic mean at $(\theta, \sigma, 0)$. This quadratic mean differentiability is established in the following lemma.

Lemma 6.1 Let $f_{1} \in \mathcal{F}_{1}, \theta \in \mathbb{R}, \sigma \in \mathbb{R}_{0}^{+}$and $\xi \in(-1,1)$. Define

$$
\begin{aligned}
g_{\theta, \sigma, \xi ; f_{1}}(x) & :=\frac{1}{\sigma} f_{1}\left(\frac{x-\theta}{\sigma(1-\operatorname{sign}(x-\theta) \xi)}\right) \\
D_{\theta} g_{\theta, \sigma, 0 ; f_{1}}^{1 / 2}(x) & :=\frac{1}{2} \sigma^{-3 / 2} f_{1}^{1 / 2}\left(\frac{x-\theta}{\sigma}\right) \phi_{f_{1}}\left(\frac{x-\theta}{\sigma}\right) \\
D_{\sigma} g_{\theta, \sigma, 0 ; f_{1}}^{1 / 2}(x) & :=\frac{1}{2} \sigma^{-3 / 2} f_{1}^{1 / 2}\left(\frac{x-\theta}{\sigma}\right)\left(\left(\frac{x-\theta}{\sigma}\right) \phi_{f_{1}}\left(\frac{x-\theta}{\sigma}\right)-1\right), \text { and } \\
\left.D_{\xi} g_{\theta, \sigma, \xi ; f_{1}}^{1 / 2}(x)\right|_{\xi=0} & :=-\frac{1}{2} \sigma^{-1 / 2} f_{1}^{1 / 2}\left(\frac{x-\theta}{\sigma}\right) \phi_{f_{1}}\left(\frac{x-\theta}{\sigma}\right)\left|\frac{x-\theta}{\sigma}\right|
\end{aligned}
$$

Then,

$$
\begin{aligned}
& \qquad\left\{g_{\theta+t, \sigma+s, r ; f_{1}}^{1 / 2}(x)-g_{\theta, \sigma, 0 ; f_{1}}^{1 / 2}(x)-\left(\begin{array}{c}
t \\
s \\
r
\end{array}\right)^{\prime}\left(\begin{array}{c}
D_{\theta} g_{\theta, \sigma, 0 ; f_{1}}^{1 / 2}(x) \\
D_{\sigma} g_{\theta, \sigma, 0 ; f_{1}}^{1 / 2}(x) \\
\left.D_{\xi} g_{\theta, \sigma, \xi ; f_{1}}^{1 / 2}(x)\right|_{\xi=0}
\end{array}\right)\right\}^{2} d x=o\left(\|(t, s, r)\|^{2}\right) \\
& \text { as }\|(t, s, r)\| \rightarrow 0 .
\end{aligned}
$$


Proof. The integral in (6.1) is bounded by $C\left(b_{1}+b_{2}+b_{3}\right)$, where $C$ is some positive constant,

$$
\begin{gathered}
b_{1}=\int\left\{g_{\theta+t, \sigma+s, r ; f_{1}}^{1 / 2}(x)-g_{\theta+t, \sigma+s, 0 ; f_{1}}^{1 / 2}(x)-\left.r D_{\xi} g_{\theta+t, \sigma+s, \xi ; f_{1}}^{1 / 2}(x)\right|_{\xi=0}\right\}^{2} d x \\
b_{2}=\int\left\{g_{\theta+t, \sigma+s, 0 ; f_{1}}^{1 / 2}(x)-g_{\theta, \sigma, 0 ; f_{1}}^{1 / 2}(x)-(t, s)\left(\begin{array}{c}
D_{\theta} g_{\theta, \sigma, 0 ; f_{1}}^{1 / 2}(x) \\
D_{\sigma} g_{\theta, \sigma, 0 ; f_{1}}^{1 / 2}(x)
\end{array}\right)\right\}^{2} d x
\end{gathered}
$$

and

$$
\begin{aligned}
b_{3} & =\int\left\{r\left(\left.D_{\xi} g_{\theta+t, \sigma+s, \xi ; f_{1}}^{1 / 2}(x)\right|_{\xi=0}-\left.D_{\xi} g_{\theta, \sigma, \xi ; f_{1}}^{1 / 2}(x)\right|_{\xi=0}\right)\right\}^{2} d x \\
& =r^{2} \int\left(\left.D_{\xi} g_{\theta+t, \sigma+s, \xi ; f_{1}}^{1 / 2}(x)\right|_{\xi=0}-\left.D_{\xi} g_{\theta, \sigma, \xi ; f_{1}}^{1 / 2}(x)\right|_{\xi=0}\right)^{2} d x .
\end{aligned}
$$

That $b_{2}$ is $o\left(\|(t, s, r)\|^{2}\right)$ is a particular case of Lemma A.1 in Hallin and Paindaveine (2006) (here in a simpler univariate context).

Turning to $b_{3}$, we only have to prove that the integral in (6.2) is $o(1)$. First note that, in view of the square-integrability of $\left.D_{\xi} g_{\theta, \sigma, \xi ; f_{1}}^{1 / 2}()\right|_{.\xi=0}$,

$$
\left\|\left.D_{\xi} g_{\theta+t, \sigma, \xi ; f_{1}}^{1 / 2}(.)\right|_{\xi=0}-\left.D_{\xi} g_{\theta, \sigma, \xi ; f_{1}}^{1 / 2}(.)\right|_{\xi=0}\right\|_{L^{2}}=\left\|\left.D_{\xi} g_{\theta, \sigma, \xi ; f_{1}}^{1 / 2}(.-t)\right|_{\xi=0}-\left.D_{\xi} g_{\theta, \sigma, \xi ; f_{1}}^{1 / 2}(.)\right|_{\xi=0}\right\|_{L^{2}}
$$

is $o(1)$ as $t \rightarrow 0$. Next, defining $f_{1 ; \exp }(x):=f_{1}\left(e^{x}\right)$ and $\left(f_{1 ; \exp }^{1 / 2}(x)\right)^{\prime}:=\frac{1}{2} f_{1}^{-1 / 2}\left(e^{x}\right) \dot{f}_{1}\left(e^{x}\right) e^{x}$, we have

$$
\begin{aligned}
& \int\left\{\left.D_{\xi} g_{\theta, \sigma+s, \xi ; f_{1}}^{1 / 2}(x)\right|_{\xi=0}-\left.D_{\xi} g_{\theta, \sigma, \xi ; f_{1}}^{1 / 2}(x)\right|_{\xi=0}\right\}^{2} d x \\
& \quad=C \int_{-\infty}^{\infty}\left(e^{\frac{1}{2}\left(u-\ln \left(1+\frac{s}{\sigma}\right)\right)}\left(f_{1 ; \exp }^{1 / 2}\right)^{\prime}\left(u-\ln \left(1+\frac{s}{\sigma}\right)\right)-e^{\frac{1}{2} u}\left(f_{1 ; \exp }^{1 / 2}\right)^{\prime}(u)\right)^{2} d u
\end{aligned}
$$

since $f_{1} \in \mathcal{F}_{1}, e^{\frac{1}{2} u}\left(f_{1 ; \exp }^{1 / 2}\right)^{\prime}(u)$ is square-integrable, so that, parallel to (6.3), quadratic mean continuity again implies that the integral in (6.4), which does not depend on $\theta$, is $o(1)$ as $s \rightarrow 0$, uniformly in $\theta$. The desired result for $b_{3}$ follows.

Finally, letting $z:=(x-\theta-t) /(\sigma+s)$, the dependence of $b_{1}$ in $(t, s)$ disappears. Quadratic mean differentiability with respect to $\xi$ then follows along the same lines as quadratic mean differentiability with respect to $\sigma$, due to the fact that $\xi$ behaves exactly as a scale parameter, except that it takes distinct values on each side of the origin.

\subsection{Asymptotic linearity.}

\subsubsection{Asymptotic linearity of $\Delta_{f_{1} ; 3}^{(n)}$ and $\Delta_{f_{1} ; 1}^{(n)}$.}

The asymptotic linearity of $\Delta_{f_{1} ; 3}^{(n)}$ in $\theta$ and $\sigma$ is required in the construction of the optimal parametric tests of Section 3.5. The proof below only requires ULAN with respect to $\theta$ and $\sigma$.

Proposition 6.1 Let $f_{1} \in \mathcal{F}_{1}$ and $g_{1} \in \mathcal{F}_{f_{1}}^{*}$. Then,

(i) for all $t \in \mathbb{R}$,

$$
\Delta_{f_{1} ; 3}^{(n)}\left(\left(\theta+n^{-1 / 2} t, \sigma, 0\right)^{\prime}\right)=\Delta_{f_{1} ; 3}^{(n)}\left((\theta, \sigma, 0)^{\prime}\right)+t \sigma^{-1} \mathcal{M}_{g_{1}}\left(f_{1}, g_{1}\right)+o \mathrm{P}(1)
$$


(ii) for all $s \in \mathbb{R}$,

$$
\Delta_{f_{1} ; 3}^{(n)}\left(\left(\theta, \sigma+n^{-1 / 2} s, 0\right)^{\prime}\right)=\Delta_{f_{1} ; 3}^{(n)}\left((\theta, \sigma, 0)^{\prime}\right)+o_{\mathrm{P}}(1)
$$

under $P_{\theta, \sigma, 0 ; g_{1}}^{(n)}$ and as $n \rightarrow \infty$.

(iii) for all $t \in \mathbb{R}$,

$$
\Delta_{f_{1} ; 1}^{(n)}\left(\left(\theta+n^{-1 / 2} t, \sigma, 0\right)^{\prime}\right)=\Delta_{f_{1} ; 1}^{(n)}\left((\theta, \sigma, 0)^{\prime}\right)-t \sigma^{-2} \mathcal{I}_{g_{1}}\left(f_{1}, g_{1}\right)+o_{\mathrm{P}}(1)
$$

(iv) for all $s \in \mathbb{R}$,

$$
\Delta_{f_{1} ; 1}^{(n)}\left(\left(\theta, \sigma+n^{-1 / 2} s, 0\right)^{\prime}\right)=\Delta_{f_{1} ; 1}^{(n)}\left((\theta, \sigma, 0)^{\prime}\right)+o_{\mathrm{P}}(1)
$$

under $P_{\theta, \sigma, 0 ; g_{1}}^{(n)}$ and as $n \rightarrow \infty$.

Proof. Similar to the proof of Proposition 5.1 in Cassart et al. (2007).

\subsubsection{Proof of Lemma 3.1.}

The proof proceeds by establishing successively that

(i) $\Delta_{f_{1} ; 3}^{*(n)}\left(\eta_{g_{1}}\left(f_{1}, g_{1}\right), \hat{\theta}_{\#}, \hat{\sigma}_{\#}\right)-\Delta_{f_{1} ; 3}^{*(n)}\left(\eta_{g_{1}}\left(f_{1}, g_{1}\right), \theta, \sigma\right)=o_{\mathrm{P}}(1)$

and

(ii) $\Delta_{f_{1} ; 3}^{*(n)}\left(\eta^{(n)}\left(\hat{\theta}_{\#}, \hat{\sigma}_{\#} ; f_{1}\right), \hat{\theta}_{\#}, \hat{\sigma}_{\#}\right)-\Delta_{f_{1} ; 3}^{*(n)}\left(\eta_{g_{1}}\left(f_{1}, g_{1}\right), \hat{\theta}_{\#}, \hat{\sigma}_{\#}\right)=o_{\mathrm{P}}(1)$, under $\mathrm{P}_{\theta, \sigma, 0 ; g_{1}}^{(n)}$, as $n \rightarrow \infty$.

Point (i) is a consequence of the asymptotic orthogonality of $\Delta_{f_{1} ; 3}^{*(n)}\left(\eta_{g_{1}}\left(f_{1}, g_{1}\right), \theta, \sigma\right)$ with $\Delta_{g_{1} ; 1}^{(n)}$ and $\Delta_{g_{1} ; 2}^{(n)}$. Integration by parts yields, for $f_{1} \in \mathcal{F}_{1}^{*}$,

$$
\mathcal{I}_{g_{1}}\left(f_{1}, g_{1}\right)=\int_{-\infty}^{\infty} \dot{\phi}_{f_{1}}(z) g_{1}(z) d z
$$

and

$$
\mathcal{M}_{g_{1}}\left(f_{1}, g_{1}\right)=\int_{-\infty}^{\infty} \operatorname{sign}(z) \phi_{f_{1}}(z) g_{1}(z) d z+\int_{-\infty}^{\infty}|z| \dot{\phi}_{f_{1}}(z) g_{1}(z) d z
$$

Thus (3.11) converges in probability to $\eta_{g_{1}}\left(f_{1}, g_{1}\right)$ under $\mathrm{P}_{\theta, \sigma, 0 ; g_{1}}^{(n)}$, as $n \rightarrow \infty$. The left hand side in (ii) can be written as

$$
T_{1}^{(n)} \times T_{2}^{(n)}:=\left(\eta^{(n)}\left(\hat{\theta}_{\#}, \hat{\sigma}_{\#} ; f_{1}\right)-\eta_{g_{1}}\left(f_{1}, g_{1}\right)\right) \times n^{-1 / 2} \sum_{i=1}^{n} \phi_{f_{1}}\left(Z_{i}^{(n)}\left(\hat{\theta}_{\#}, \hat{\sigma}_{\#}\right)\right) .
$$

Le Cam's third Lemma implies that $T_{1}^{(n)}$ is $o_{\mathrm{P}}(1)$ under $\mathrm{P}_{\theta, \sigma, 0 ; g_{1}}^{(n)}$ as soon as the asymptotic covariances of $\mathcal{I}^{(n) *}\left(\theta, \sigma ; f_{1}\right)$ and $\mathcal{M}^{(n) *}\left(\theta, \sigma ; f_{1}\right)$ with $\Delta_{g_{1} ; 1}^{(n)}$ and $\Delta_{g_{1} ; 2}^{(n)}$ are finite. A simple computation shows that this is indeed the case under (3.12) and (3.13). Now, ULAN implies that

$$
T_{2}^{(n)}=n^{-1 / 2} \sum_{i=1}^{n} \phi_{f_{1}}\left(Z_{i}^{(n)}(\theta, \sigma)\right)+\left(\sigma^{-1} \mathcal{I}_{g_{1}}\left(f_{1}, g_{1}\right) 0\right) n^{1 / 2}\left(\left(\begin{array}{c}
\hat{\theta}_{\#} \\
\hat{\sigma}_{\#}
\end{array}\right)-\left(\begin{array}{c}
\theta \\
\sigma
\end{array}\right)\right)+o_{\mathrm{P}}(1),
$$

as $n \rightarrow \infty$ under $\mathrm{P}_{\theta, \sigma, 0 ; g_{1}}^{(n)}$. Hence, the central limit theorem and root- $n$ consistency of $\hat{\theta}_{\#}$ and $\hat{\sigma}_{\#}$ entail that (6.5) is $O_{\mathrm{P}}(1)$. 


\subsubsection{Asymptotic linearity of the signed-rank version of the central sequence.}

Proposition 6.2 Let $f_{1} \in \mathcal{F}_{1}^{\square}$ and $g_{1} \in \underset{\sim}{\mathcal{F}_{f_{1}}^{*}}$. Then, for all $t \in \mathbb{R}$,

$$
\underset{\sim}{\Delta} f_{1 ; 3}^{(n)}\left(\theta+n^{-1 / 2} t\right)=\underset{\sim}{\Delta} f_{1 ; 3}^{(n)}(\theta)+t \sigma^{-1} \mathcal{M}\left(f_{1}, g_{1}\right)+o_{\mathrm{P}}(1)
$$

as $n \rightarrow \infty$ under $\mathrm{P}_{\theta, \sigma, 0 ; g_{1}}^{(n)}$.

Proof. Van Eeden (1972)'s Theorem 3.2 applies, yielding the desired result.

\section{References}

[1] Antille, A. (1974). A linearized version of the Hodges-Lehmann estimator. Annals of Statistics 2, 1308-1313.

[2] Arellano-Valle, R.B., Gómez, H.W. and Quintana, F.A. (2005). Statistical inference for a general class of asymmetric distributions. Journal of Statistical Planning and Inference 128, 427-443.

[3] Azzalini, A. and Capitanio, A. (2003). Distributions generated by perturbation of symmetry with emphasis on a multivariate skew $t$ distribution. Journal of the Royal Statistical Society Series B 65, 367-389.

[4] Beran, R. (1974). Asymptotically efficient adaptive rank estimates in location models. Annals of Statistics 2, 63-74.

[5] Bickel, P.J. and Ritov, Y. (1988). Estimating integrated squared density derivatives. Sankhya A 50, 381-393.

[6] Butler, C.C. (1969). A test for symmetry using the sample distribution function. The Annals of Mathematical Statistics 40, 2209-2210.

[7] Cassart, D., Hallin, M. and Paindaveine, D. (2007). A class of optimal tests for symmetry based on Edgeworth approximations. Submitted.

[8] Cheng, K.F. and Serfling, R.J. (1981). On estimation of a class of efficiency-related parameters. Scandinavian Actuarial Journal 8, 83-92.

[9] Fan, J. (1991). On the estimation of quadratic functionals. Annals of Statistics 19, 12731294.

[10] Fechner, G.T. (1897). Kollectivmasslehre. Engleman, Leipzig.

[11] Hallin, M., Oja, H., Paindaveine, D. (2006). Semiparametrically efficient rank-based inference for shape: II Optimal R-estimation of shape. Annals of Statistics 34, 2757-2789.

[12] Hallin, M. and Paindaveine, D. (2006). Semiparametrically efficient rank-based inference for shape: I Optimal rank-based tests for sphericity. Annals of Statistics 34, 2707-2756.

[13] Hallin, M. and Werker, B.J.M. (2003). Semiparametric efficiency, distribution-freeness, and invariance. Bernoulli 9, 137-165. 
[14] Hill, D.L. and Rao, P.V. (1977). Tests of symmetry based on Cramér-von Mises statistics. Biometrika 64, 489-494.

[15] Jurečková, J. and Sen, P.K. (1996). Robust Statistical Procedures : Asymptotics and Interrelations. New York: Wiley.

[16] Koul, H.L. (2002). Weighted Empirical Processes in Dynamic Nonlinear Models, 2nd edition. New York: Springer Verlag.

[17] Kraft, C.H. and van Eeden, C. (1972). Linearized rank estimates and signed-rank estimates for the general linear hypothesis. The Annals of Mathematical Statistics 43, 42-57.

[18] Kreiss, J-P. (1987). On adaptative estimation in stationary ARMA processes. Annals of Statistics 15, 112-133.

[19] Le Cam, L.M. (1986). Asymptotic Methods in Statistical Decision Theory. New-York: Springer-Verlag.

[20] Lehmann, E.L. (1963). Nonparametric confidence intervals for a shift parameter. The Annals of Mathematical Statistics 34, 1507-1512.

[21] McWilliams, T.P. (1990). A distribution-free test for symmetry based on a runs statistic. Journal of the American Statistical Association 85, 1130-1133.

[22] Puri, M.L. and Sen, P.K. (1985). Nonparametric Methods in General Linear Models. New York : J. Wiley.

[23] Randles, R.H., Fligner, M.A., Policello, G.E. and Wolfe, D.A. (1980). An asymptotically distribution-free test for symmetry versus asymmetry. Journal of the American Statistical Association 75, 168-172.

[24] Rothman, E.D. and Woodrofe, M. (1972). A Cramér-von Mises type statistic for testing symmetry. The Annals of Mathematical Statistics 43, 2035-2038.

[25] Schweder, T. (1975). Window estimation of the asymptotic variance of rank estimators of location. Scandinavian Journal of Statistics 2, 113-126.

[26] Sen, P.K. (1966). On a distribution-free method of estimating asymptotic efficiency of a class of nonparametric tests. The Annals of Mathematical Statistics 37, 1759-1770.

[27] Stein, C. (1956). Efficient nonparametric testing and estimation. Proceedings of the Third Berkeley Symposium on Mathematical Statistics and Probability 1, 187-196.

[28] Stone, C. (1975). Adaptive maximum likelihood estimation of a location parameter. Annals of Statistics 3, 267-284.

[29] Swensen, A. R. (1985). The asymptotic distribution of the likelihood ratio for autoregressive time series with a regression trend. Journal of Multivariate Analysis 16, 54-70.

[30] Van Eeden, C. (1972). An analogue, for signed-rank statistics, of Jurečková's asymptotic linearity theorem for rank statistics. The Annals of Mathematical Statistics 43, 791-802. 